\title{
Your reviews or mine? Exploring the determinants of "perceived helpfulness" of online reviews: a cross-cultural study
}

\author{
Baidyanath Biswas $^{1}$ (I) Pooja Sengupta ${ }^{2} \cdot$ Boudhayan Ganguly $^{1}$
}

Received: 19 May 2020 / Accepted: 7 December 2020 / Published online: 15 January 2021

(C) Institute of Applied Informatics at University of Leipzig 2021

\begin{abstract}
E-commerce platforms allow customers to post online reviews about their products, but many of these reviews remain non-voted by existing customers. Current literature on the perceived helpfulness of online reviews has overlooked the effect of these non-voted reviews. Additionally, current studies lack the cross-cultural perspective to help analyze the influence of cultural factors during an analysis of online reviews in an e-commerce platform. This study proposes a novel cross-cultural framework using online consumer reviews from a global e-commerce retailer to investigate these shortcomings. Our study has twofold contributions: firstly, we identified the significant predictors (such as review-title, review sentiments, star rating, social context, and temporal features) of the count of helpful votes received by online reviews - both voted and non-voted. Secondly, we identified a strong moderating effect of national culture on these predictors during the perception and evaluation of global consumers' reviews. We also performed a quantile regression across the entire distribution of the count of helpful votes. Findings from our study will guide reviewers who publish helpful and comprehensive reviews. Additionally, customers and sellers from global e-commerce firms will learn to account for cross-cultural differences on the provided features to prevent the distortion of the information content of online reviews.
\end{abstract}

Keywords Online consumer reviews $\cdot$ Perceived helpfulness ·Zero-inflated poisson distribution $\cdot$ Quantile regression $\cdot$ Hofstede's cultural framework

JEL classification $\mathrm{C} 21 \cdot \mathrm{L} 81$

Responsible Editor: Makoto Nakayama

Baidyanath Biswas

b.biswas@imi-k.edu.in

Pooja Sengupta

p.sengupta@imi-k.edu.in

Boudhayan Ganguly

b.ganguly@imi-k.edu.in

1 Information Technology Area, International Management Institute, 2/4 C, Judges Ct Rd, Alipore, Kolkata 700027, India

2 Quantitative Techniques Area, International Management Institute, 2/4 C, Judges Ct Rd, Alipore, Kolkata 700027, India

\section{Introduction and motivation}

The Internet has enabled businesses to connect with their customers through electronic marketplaces and subsequently sell their products and services. Customers use these emarketplaces to collect relevant information about the products and services, make purchase decisions, and share their assessments as user-generated content (UGC) in the form of online consumer reviews (OCR). These OCRs are perceived to be more user-oriented than traditional marketing communication, as they describe the products precisely in terms of various usage scenarios and assess them from the perspective of peer users (Filieri et al. 2018). Additionally, e-businesses are highly dependent on OCRs because they influence the future sale of products and services. The Spiegel Research Center at Northwestern University found that the likelihood of purchase for products with at least five reviews is $270 \%$ 
greater than those products with no available reviews. ${ }^{1}$ Current academic literature has also conclusively established the impact of OCRs on the subsequent sale of products in electronic markets (Ghose and Ipeirotis 2010; Erkan and Evans 2016; Babić Rosario et al. 2016; Ismagilova et al. 2019; Li et al. 2019a, b, 2020; Ismagilova et al. 2020).

Often, consumers vote for an OCR as helpful when they find it useful and trustworthy during the information search, thereby reducing the uncertainty associated with the final purchase decisions. Mudambi and Schuff (2010) define "a helpful customer review as a peer-generated product evaluation that facilitates the consumer's purchase decision process." However, it is challenging to assess reviews that have not received any helpful votes (Ghose and Ipeirotis 2010; Chua and Banerjee 2016; Qazi et al. 2016). Therefore, it becomes imperative to understand the determinants of the perceived helpfulness of OCRs, taking into account the fact that some of them are displayed on the e-commerce platforms without any helpful votes.

Further, consumers from different countries are not a monolithic group. So, the effect of perceived helpfulness of reviews and its impact on a consumer's buying decisions may vary across different national cultures while referring to previous UGCs. Recently, many e-businesses have begun to operate across multiple countries (Agarwal and Wu 2018) and cater to customers from different cultural backgrounds ${ }^{2,3,4}$ Scholars acknowledge that the diverse cultural background of these users imparts varying levels of emphasis on the features of an e-commerce platform, information about the listed products, and UGCs (Hong et al. 2016; Tang 2017; Zhu et al. 2017; Gao et al. 2018; Rosillo-Díaz et al. 2019; Stamolampros et al. 2019). Recently, Amazon India launched "Global Stores," where Indian sellers and businesses showcase their products and sell to international users from North America, Europe, and Australia. ${ }^{5}$ Therefore, prospective buyers from these countries now refer to reviews from Amazon India that were written by Indian users. These cross-cultural scenarios create a possible moderating effect (Tang 2017; Stamolampros et al. 2019; Prince et al. 2020) during the perception and evaluation of OCRs (Nakayama and Wan 2018, 2019a, b). Sometimes, these platforms continue to use universally standardized algorithms without incorporating cultural elements, even leading to strategic failures. ${ }^{6}$ Thus, we pose

\footnotetext{
1 "How Online Reviews Influence Sales" by Spiegel Research Center (2017): https://spiegel.medill.northwestern.edu/_pdf/Spiegel_Online\%20Review_ eBook Jun2017 Pv2.pdf

${ }^{2}$ EBay Global Store: https://www.ebay.com/str/onlineglobalstore

${ }^{3}$ Etsy Global Store: https://www.etsy.com/market/global

${ }^{4}$ Sell on Alibaba Globally: https://seller.alibaba.com/?country=US

${ }^{5}$ Amazon Global Selling: https://services.amazon.in/services/amazon-globalselling/benefits.html

${ }^{6}$ Amazon admits defeat against Chinese e-commerce rivals like Alibaba and JD.com: https://www.theverge.com/2019/4/18/18485578/amazon-chinamarketplace-alibaba-jd-e-commerce-compete
}

our first research question: RQ1: How does national culture affect the relationship among the determinants of the count of helpful votes for online consumer reviews?

Methodologically, advances in the current literature on the perceived helpfulness of OCRs are limited as they examined count of helpful votes as (i) a binary variable - helpful or not helpful (Mudambi and Schuff 2010; Agnihotri and Bhattacharya 2016; Chua and Banerjee 2016; Qazi et al. 2016); (ii) a continuous variable (Salehan and Kim 2016), or (iii) rejected OCR observations that did not receive any helpfulness votes (Chua and Banerjee 2015; Kuan et al. 2015; Salehan and Kim 2016; Malik and Hussain 2017). However, such a subjective treatment of OCRs completely ignores the true nature of the helpful votes, which is countdata. It also drastically reduces the power of the final empirical models. Further, we are interested to know how the determinants of OCRs affect the perceived helpfulness across the entire distribution of the count of helpful votes. Hence, we pose our second research question: RQ2: What are the significant determinants of the count of helpful votes for online consumer reviews?

To address these research questions, we propose a theoretical framework to analyze the influence of national culture on OCRs. First, we executed a web-scraper in R to extract the OCR data for an assortment of products from Amazon India and the USA. Second, we applied advanced data-analytics (Akter and Wamba 2016) and text mining to the extracted data, segregated them in terms of OCR features supported by the Theory of Reasoned Action, Social Presence Theory, and the Self-Efficacy Theory. Third, we identified the crosscultural influence of those predictors on the perceived helpfulness of OCRs and measured them quantitatively. Our study will enable e-commerce platforms and sellers to acknowledge the features that influence global consumers while making purchasing decisions using both voted and non-voted reviews. As a result, these platforms will gain strategic knowledge (Alt and Zimmermann 2019) about the factors that make a review helpful and subsequently apply them to predict nonvoted reviews, rank them, improve product visibility, and the displayed product-information on the e-marketplace. Therefore, in this study, we identified cross-cultural influences on OCRs and measured them quantitatively.

Figure 1 illustrates a typical online review displayed on Amazon India, highlighted with the associated characteristics that serve as the predictors of the count of helpful votes in our study.

The remainder of this paper is organized as follows. In the next few sections, we present an overview of the literature on the perceived helpfulness of OCRs and relevant behavioral theories. Then, we build hypotheses and describe the conceptual framework. Next, we outline the research methodology, describe the data, generate the results, and discuss them. Then, we report the implications of our study towards extant theories 


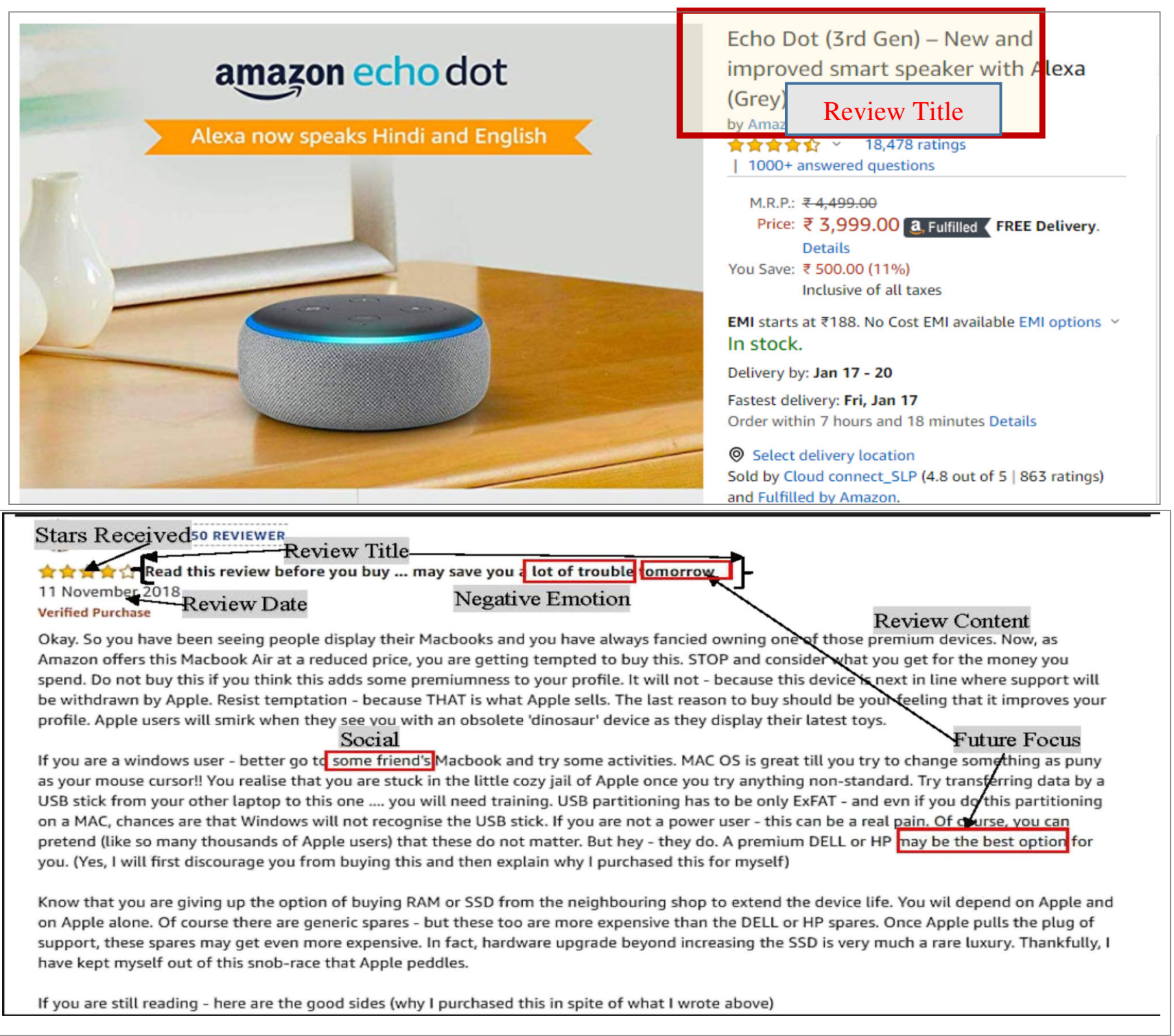

Fig. 1 A typical online review and its associated features (from Amazon India)

and managerial practices. Finally, we offer future research directions and conclude the study.

\section{Literature review}

The extant literature on the perceived helpfulness of online consumer reviews reports that OCRs help customers to acquire relevant and timely information about a product or service on the e-commerce platform, thereby reducing the information asymmetry between the seller and the buyer (Liu et al. 2017; Ismagilova et al. 2019, 2020). This attribute makes an OCR helpful to a prospective online customer and positively affects future sales. Broadly, the current literature on OCRs have focussed upon two aspects: Type I: determinants of perceived helpfulness of OCRs, and Type II: the effect of OCRs on the subsequent sale of products on the online platforms. Meta-analyses on Type I (Hong et al. 2017; Wang et al. 2019) and Type II (Babić Rosario et al. 2016; Ismagilova et al. 2019; $\mathrm{Li}$ et al. 2020) are available in the current literature. In this section, we briefly explore and discuss the current state of the literature on Type I.

Most e-commerce platforms ask their online users: "whether this review was helpful to you?" to which the user answers as "yes" or "no." If they mark the question as "yes," it counts towards the perceived helpfulness votes received by the OCR. Multiple studies have examined the effect of review readability, spelling errors, average helpfulness of a reviewer's historical reviews, level of subjectivity in the expressions used to write reviews, and the reviewer's history to predict the count of helpful votes. They have examined OCR data from Amazon. com across different categories of products with the help of 
OLS regression as well as Support Vector Machines and Random Forests, leading to three types of predictors: reviewer-based, OCR subjectivity, and OCR readability.

Mudambi and Schuff (2010) examined review data across six products from Amazon.com with Tobit Regression to study the effect of review ratings, review extremity, and review depth on the products' helpfulness votes, which were moderated by the product-type. Next, using review data for software downloaded from CNET.com, Cao et al. (2011) investigated why the majority OCRs remained without any helpful votes, while only a few received a high number of votes. They applied the following review characteristics: (i) basic (i.e., date, extremity, subjectivity), (ii) stylistic (i.e., the average number of words and characters used in the title and the review-text), and (iii) semantic (i.e., the meaning of the words used in the review-text) suggesting that semantic features influenced most users to vote OCRs as helpful.

Li and Zhan (2011) identified different predictors of perceived helpfulness of OCRs such as language style, organizational structure, and other textual features from Amazon reviews. Racherla and Friske (2012) employed different features such as days elapsed, reviewer's identity disclosure, reviewer expertise, reputation, extensiveness (measured by the count of words), and valence (measured by star ratings). They studied the effects of these predictors on the review helpfulness for service apps extracted from Yelp.com. While examining the contribution of style and content of e-WOMs toward their helpfulness, Schindler and Bickart (2012) found that the review-length exerted a positive effect on the perceived helpfulness up to a threshold, while the spelling and grammatical errors were associated with less helpful reviews.

Using Amazon.com data, Baek et al. (2012) applied dualprocessing theories and found that both peripheral cues (i.e., review rating and reviewer's credibility) and central cues (i.e., the content of reviews) were responsible for the perceived helpfulness votes. Based on bulletin-board reviews, Li et al. (2013) conducted a laboratory experiment to consider source credibility, content diagnosticity, and vicarious expressions used in OCRs to identify the perceived helpfulness as a second-order construct. Flanagin and Metzger (2013) also found that source and content-based features directly affected the review's perceived helpfulness, and with customer-written OCRs emerged more helpful than expert-written ones.

Next, Huang et al. (2015) employed a set of qualitative (i.e., reviewer experience, reviewer impact, cumulative helpfulness, and product rating) and quantitative (i.e., word count) features. They studied the effect of those features on the review helpfulness, revealing a threshold for word counts. Drawing from information-processing theories, Kuan et al. (2015) demonstrated the effect of review content (i.e., wordcount, readability), review valence (i.e., count of positive and negative-loaded words), and review extremity (i.e., rating difference), and reviewer credibility (whether top reviewer) on review voting and perceived review helpfulness. They found that not all OCRs were equally likely to receive helpfulness votes and that systematic differences existed between these two groups. Chua and Banerjee (2015) examined the review helpfulness as a function of reviewer reputation (i.e., reviewer rankings) and review ratings (i.e., stars received), which were moderated by review depth (i.e., count of words in review-text). They found that users voted for negative OCRs among low-depth reviews, while they reacted skeptically for high-depth reviews. Salehan and Kim (2016) explored the predictors of OCRs' readership and helpfulness using sentiment-mining (Liu 2012) to find that OCRs with neutral polarity were perceived to be more helpful than highly sentimental reviews.

Recently, Agnihotri and Bhattacharya (2016) proposed qualitative cues (such as review readability, positive and negative sentiments) in addition to quantitative cues (such as star ratings) that followed an ideal point concept, such that the perceived helpfulness decreased after a certain point. Lee and Choeh (2016) demonstrated that the reviewer's reputation, identity, and review depth could influence an OCR's helpfulness. Additionally, they observed a moderating effect for various product types across these predictors.

$\mathrm{Fu}$ et al. (2017) tested the mediation effects of review ratings, the delay between memberships, and subsequent review helpfulness to find that review depth had a positive effect on review helpfulness, while review ratings reflected customer attitudes and affective inclinations toward the sellers. On the contrary, Ghasemaghaei et al. (2018) examined online consumer reviews for automobile, home, and life insurance products and found that longer reviews received lesser ratings due to negativity bias.

Based on the extant studies, we categorized the relevant literature on the perceived helpfulness of online consumer reviews into three primary streams: review-based, reviewerbased, and user-based, their associated variables, and methodologies, and present them in Table 1.

\section{Theoretical development}

Our study draws from the following theories on "informational influence" that elucidate the mechanisms of influencing individuals through the information that they receive (Sussman and Siegal 2003). These are Theory of Reasoned Action, Social Presence Theory, and Self-Efficacy Theory, all of which help us examine the effects of review characteristics on its perceived helpfulness in an e-commerce platform. Hofstede's Cultural Framework guides our cross-cultural examination of the OCRs and their relationship with perceived helpfulness. 
Table 1 Summary of current literature on the perceived helpfulness of online consumer reviews

\begin{tabular}{|c|c|c|c|c|}
\hline & Factors & Details & Methodology & Academic sources \\
\hline \multirow[t]{12}{*}{ Review-based } & \multirow[t]{2}{*}{ Title } & Depth (length) of title & TR, NB & Qazi et al. (2016); Salehan and Kim (2016) \\
\hline & & Sentiments/polarity & NB & Salehan and Kim (2016) \\
\hline & \multirow[t]{9}{*}{ Content } & Depth (length) of review text & TR, NB & $\begin{array}{l}\text { Mudambi and Schuff (2010); Schindler and Bickart } \\
\text { (2012); Chua and Banerjee (2015); Lee and Choeh } \\
\text { (2016); Ngo-Ye et al. (2017); Guo and Zhou (2017); } \\
\text { Ghasemaghaei et al. (2018) }\end{array}$ \\
\hline & & $\begin{array}{l}\text { Sentiments/polarity, positive and } \\
\text { negative words, } \\
\text { squared-sentiments, } \\
\text { sentiment-deviation }\end{array}$ & TR, TM, SVR & $\begin{array}{l}\text { Baek et al. (2012); Schindler and Bickart (2012); Yin et al. } \\
\text { (2014); Ahmad and Laroche (2015); Salehan and Kim } \\
\text { (2016); Agnihotri and Bhattacharya (2016); } \\
\text { González-Rodríguez et al. (2016); Ngo-Ye et al. (2017); } \\
\text { Ghasemaghaei et al. (2018); Fresneda and Gefen (2019); } \\
\text { Ismagilova et al. (2020) }\end{array}$ \\
\hline & & Semantics & TR, TM, SVR & Cao et al. (2011); Guo and Zhou (2017); \\
\hline & & $\begin{array}{l}\text { Stylistic elements, spelling and } \\
\text { grammatical errors, lexical } \\
\text { density }\end{array}$ & $\begin{array}{l}\mathrm{RF}, \mathrm{NaB}, \mathrm{HR} \\
\quad \mathrm{NB}\end{array}$ & $\begin{array}{l}\text { Forman et al. (2008); Ghose and Ipeirotis (2010); Cao } \\
\text { et al. (2011); Baek et al. (2012); Schindler and Bickart } \\
\text { (2012); Ahmad and Laroche (2015); Krishnamoorthy } \\
\text { (2015); Chua and Banerjee (2016) }\end{array}$ \\
\hline & & Readability & TR, NB, OLS & $\begin{array}{l}\text { Forman et al. (2008); Ghose and Ipeirotis (2010); } \\
\text { Krishnamoorthy (2015); Agnihotri and Bhattacharya } \\
\text { (2016); Chua and Banerjee (2016); Ngo-Ye et al. } \\
\text { (2017); Malik and Hussain (2017); Fresneda and Gefen } \\
\text { (2019) }\end{array}$ \\
\hline & & Age & $\begin{array}{l}\text { OLS,TM, SVR, } \\
\text { RF, NaB }\end{array}$ & $\begin{array}{l}\text { Racherla and Friske (2012); Krishnamoorthy (2015); } \\
\text { Salehan and Kim (2016); Ngo-Ye et al. (2017); Guo and } \\
\text { Zhou (2017) }\end{array}$ \\
\hline & & Analytical Thinking & ZIP & This Study \\
\hline & & Social Connection & ZIP & This Study \\
\hline & & Temporal focus & ZIP & This Study \\
\hline & $\begin{array}{l}\text { Review Ratings } \\
\quad \text { (valence) }\end{array}$ & $\begin{array}{l}\text { Overall ratings, rating } \\
\text { inconsistency, extremity, } \\
\text { squared-ratings }\end{array}$ & TM, SVR, HR & $\begin{array}{l}\text { Mudambi and Schuff (2010); Baek et al. (2012); Huang } \\
\text { et al. (2015); Lee and Choeh (2016); Ngo-Ye et al. } 2017 \\
\text { Guo and Zhou (2017); Wang et al. (2019); } \\
\text { Ghasemaghaei et al. (2018); Wang and Karimi (2019) }\end{array}$ \\
\hline \multirow[t]{3}{*}{ Reviewer-based } & \multirow[t]{3}{*}{$\begin{array}{l}\text { Reviewer } \\
\text { Information }\end{array}$} & $\begin{array}{l}\text { Identity disclosure, profile photo, } \\
\text { real name }\end{array}$ & OLS, TR, NB & $\begin{array}{l}\text { Ghose and Ipeirotis (2010); Racherla and Friske (2012); } \\
\text { Liu and Park (2015); Agnihotri and Bhattacharya } \\
\text { (2016); Lee and Choeh (2016) }\end{array}$ \\
\hline & & Reputation, Expertise, credibility & OLS, NB & $\begin{array}{l}\text { Ghose and Ipeirotis (2010); Racherla and Friske (2012); } \\
\text { Chua and Banerjee (2015); Chua and Banerjee (2016); } \\
\text { Lee and Choeh (2016); Guo and Zhou (2017) }\end{array}$ \\
\hline & & $\begin{array}{l}\text { Commitment, current activity, } \\
\text { social network }\end{array}$ & $\begin{array}{l}\text { OLS, TM, TR, } \\
\text { SVR, HR }\end{array}$ & $\begin{array}{l}\text { Racherla and Friske (2012); Baek et al. (2012); Ngo-Ye } \\
\text { and Sinha (2014); Agnihotri and Bhattacharya (2016); } \\
\text { Lee and Choeh (2016); Malik and Hussain (2017) }\end{array}$ \\
\hline \multirow[t]{2}{*}{ User-based } & \multirow[t]{2}{*}{$\begin{array}{l}\text { Cross-cultural } \\
\text { influences }\end{array}$} & Hofstede's Culture Framework & Text Analytics & $\begin{array}{l}\text { Nakayama and Wan (2018); Nakayama and Wan (2019a); } \\
\text { Nakayama and Wan (2019b); Stamolampros et al. } \\
\text { (2019) }\end{array}$ \\
\hline & & & ZIP & This Study \\
\hline
\end{tabular}

OLS Ordinary Least-Square Regression, NB Negative Binomial Regression, TR Tobit Regression, ZIP Zero-Inflated Poisson Regression, TM TextMining, SVR Support Vector Regression, $R F$ Random Forests, $N a B$ Naïve Bayes, HR Hierarchical Regression

\section{Theory of reasoned action}

Fishbein and Ajzen (1975) proposed the Theory of Reasoned Action (TRA) to explain the behavioural intentions that lead to a particular action by an individual. Among other technology adoption theories, TRA regards that an individual's intention precedes its behavioural action(s), and thereby, TRA helps to identify the factors that directly influence the perceived helpfulness of OCRs. This intention is determined by (i) the attitude towards the exhibited behaviour, (ii) related social stimuli, and 
(iii) motivation to respond to those stimuli. (Wu and Liu 2007; Erkan and Evans 2016). Using TRA, Lee and Shin (2014) examined the quality of OCRs and their influence on consumers' purchase intention to identify that review quality exerted significant influence on the purchase intention for a search good but had dual effects for an experience good. Lo and $\mathrm{Qu}(2015)$ predicted the tourists' intention to visit and shop in a destination, and with external variables drawn from TRA: quality, value, and satisfaction. Using TRA, Erkan and Evans (2016) examined the influence of UGCs in social media on consumers' purchase intentions. Pai and Liu (2018) applied TRA for Twitter data and stock market indices using timeseries models to predict vehicle sales. Guided by TRA, ElSaid (2020) examined the direct influence of OCRs on the user's intention of hotel-booking using the brand image, hotel star category, and price importance as moderators. Therefore, a prospective user may be motivated after reading the review, which in turn may affect his attitude towards the product, leading to better decision-making before purchase, and thereby marking the review as "helpful." Based on TRA, we posit that the following determinants of an OCR: (i) review title-based: length, positive and negative sentiments, (ii) review contentbased: positive and negative sentiments, can explain its perceived helpfulness for peer users.

\section{Social presence theory}

Short et al. (1976) proposed Social Presence Theory (SPT) to deal with the differences in the physical proximity generated by various communication media. Media plays a vital role in creating the requisite proximity between users during online information assimilation. Scholars conceptualized social presence as the sense of human warmth, and sociability conveyed through OCRs in an e-commerce platform (Zhang et al. 2014; Lu et al. 2016). Recently, SPT has received considerable attention in UGC literature since it explains the consumer-behaviour on social commerce platforms manifested through peer-interaction with shared artifacts (Friedrich et al. 2019). These platforms often incorporate several communication cues, such as facial expressions, gestures, and textual media, to generate a high degree of social presence (Hernández-Ortega 2018; Srivastava and Chandra 2018). Gradually, when the social presence is high, a prospective user perceives distant entities (such as OCRs submitted by unknown users) to be close, thereby increasing the psychological proximity (Choi et al. 2001; Xu 2014).

Additionally, social presence may also represent users' experiences within the e-commerce platform (Zhao et al. 2018). For instance, when there are multiple system features to help the consumers evaluate reviews and associated reviewers, they perceive the platform as considerate about their needs. Therefore, such a social presence can generate a more warm and harmonious feeling among users who are more likely to perceive that the displayed reviews are dependable and helpful (Xu 2014; Hernández-Ortega 2018; Liu et al. 2020). Further, they are more likely to generate a sense of contact and enhance their evaluation of products and services (Zhou et al. 2015). Thus, SPT can successfully explain the perceived helpfulness of online consumer reviews through an increased social presence. In this study, we posit, based on SPT, that the following determinants of an OCR, namely, analytical content, readability, grammatical completeness, and socially relevant content, can explain its perceived helpfulness for peer users through an increased social presence.

\section{Self-efficacy theory}

Bandura (1977) proposed the Self Efficacy Theory (SET), which considered an individual's ability to perform tasks without external guidance. Self-efficacy indicates whether an individual is skilled enough to accomplish a task in a specific method or achieve a predefined goal. Often a prior belief exists that an individual possesses the competencies to implement the courses of action necessary to cope with the forthcoming circumstances. Users generally engage in tasks where their self-efficacy is high and avoid those tasks where their self-efficacy is low. Often, self-efficacy reduces the perceived risk of online transactions, increases trust with the seller, and makes an online consumer review perceived as "helpful" (Kim and Kim 2005; Dash and Saji 2008).

Applying SET in e-commerce, Hocevar et al. (2014) observed that social media users, who are high on self-efficacy, relied more on others' opinions, specifically when evaluating the information. Besides, Furner et al. (2018) showed that selfefficacy directly affected the information quality and increased trust and purchase intention across m-commerce users. Park et al. (2020) examined different messages posted at online healthcare communities to find that high self-efficacy messages positively affected their helpfulness. Thus, the perceived helpfulness of existing online consumer reviews influences users' self-efficacy, making better purchase decisions. In this study, SET guides us to build the following predictors of perceived helpfulness for online consumer reviews: present temporal focus and future temporal focus. These predictors positively affect the information quality and improve the user's purchase intention, thereby rendering the review as "helpful."

\section{Hofstede's framework: Culture and its influence on online reviews}

Culture is a multi-dimensional construct, and its effect across online media has been extensively studied in the literature (Cyr 2008; Ganguly et al. 2010; Gunkel et al. 2014; Shiu 
et al. 2015; Cyr et al. 2017; Tang 2017). According to Kroeber and Kluckhohn (1952), "culture consists of patterns, explicit and implicit, of and for behaviour acquired and transmitted by symbols, constituting the distinctive achievements of human groups, including their embodiments in artifacts." Again, Kluckhohn and Kelly (1945) have said, "culture is historically created designs for living." According to Hofstede et al. (1991), culture is the "collective programming of the mind which distinguishes the members of one group or category of people from another." Despite criticisms (McSweeney 2002) regarding the validity of Hofstede's works (Hofstede 1984; Hofstede et al. 1991; Minkov and Hofstede 2012); they have been applied and validated in numerous cross-cultural studies for online consumer reviews (Hong et al. 2016; Tang 2017; Zhu et al. 2017; Nakayama and Wan 2018, 2019a, b; Stamolampros et al. 2019).

\section{Hofstede's dimensions of culture}

Hofstede (1984) defined culture using four primary dimensions: power distance, uncertainty avoidance, masculinityfemininity, and collectivism-individualism. Later, Hofstede et al. (1991) proposed long-term vs. short-term orientation as the fifth dimension in the existing framework. First, power distance refers to the ability of the members of a culture to accept the unequal distribution of power. Next, uncertainty avoidance relates to the ability of the members of a culture to avoid unknown and ambiguous situations. Masculinity refers to the extent of assertiveness, display of success, and achievement in a society, whereas femininity symbolizes qualities of life and feelings for others. Next, collectivism imparts more importance to group-welfare and family values than individualism, which derives from personal achievements and self-centered roles. Long-term orientation enables the members of a culture to focus on future goals rather than a short-term orientation that deals with immediate outcomes and quick material gains. We chose the USA and India for our analysis, as they have diverse scores for these cultural dimensions, which are presented in Table 2.

\section{Conceptual framework and hypothesis development}

\section{Effect of review title characteristics on helpfulness}

In e-commerce platforms, reviews are a form of UGC and typically composed and delivered in textual format (Huang et al. 2015). Although the entire OCR can provide valuable information to prospective consumers during the purchasing decision, perceived helpfulness can widely vary with the title's word count (Qazi et al. 2016; Salehan and Kim 2016). For instance, a review title "The sound quality was not good. My laptop started giving issues with the power button ..." is more helpful to a consumer than a title simply saying "Superb." Hence, we expect the title length to be positively related to its perceived helpfulness.

The title is an essential component of a review and provides new users with a glimpse of the general theme (Wang and Karimi 2019). Thus, the title-text can effectively communicate the sentiment (Liu 2012) and significantly influence the reader (Salehan and Kim 2016). Besides, we observe that a review's associated affective language receives more attention than the one written in a neutral expression (Chua and Banerjee 2016; Ismagilova et al. 2020). Hence, a review title's positive and negative sentiments can positively affect its perceived helpfulness (Yin et al. 2014; Agnihotri and Bhattacharya 2016). Therefore, we hypothesize:

Hypothesis H1a: The length of a review title has a positive effect on its perceived helpfulness.

Hypothesis H2a: The positive sentiment of a review title has a positive effect on its perceived helpfulness.

Hypothesis H3a: The negative sentiment of a review title has a positive effect on its perceived helpfulness.

\section{Cultural influence on review title characteristics}

Hofstede (1984) noted that users from high masculinity cultures are high on assertiveness and emphasize unequivocal decision-making. Again, feminine cultures are more of a
Table 2 Country scores of USA and India along the cultural dimensions of Hofstede

\begin{tabular}{llllll}
\hline Country & $\begin{array}{l}\text { Power } \\
\text { distance }\end{array}$ & $\begin{array}{l}\text { Uncertainty } \\
\text { avoidance }\end{array}$ & $\begin{array}{l}\text { Masculinity } \\
\text { vs. Femininity }\end{array}$ & $\begin{array}{l}\text { Individualism vs. } \\
\text { Collectivism }\end{array}$ & $\begin{array}{l}\text { Long term vs. } \\
\text { Short term }\end{array}$ \\
\hline USA & 40 & 46 & 62 & 91 & 26 \\
India & 77 & 40 & 56 & 48 & 51 \\
\hline
\end{tabular}

Source: Based on Hofstede (1984); Hofstede et al. (1991); Minkov and Hofstede (2012) 
thinking and observing type than masculine cultures, who are more of a doing and acquiring type (Cyr 2008; Cyr et al. 2017; El Ghoul and Zheng 2016). This type of userbehaviour relates to active information search about a product and its subsequent purchase. Users hailing from highly masculine societies look for those attributes in the review-title, which can help them make quicker purchasing decisions and prefer reading shorter review-titles with more direct and unambiguous information. On the other hand, users from highly feminine societies are more of a thinking and observing type and therefore wish to see reasonably longer review-titles that can help them acquire more stimulating insinuations about the product. Thus, the USA users, being high on masculinity than Indian users, will prefer focussed, short yet expressive reviewtitles that can quickly convey product-related information. And Indian users being low on masculinity than the USA users, will impart more importance on the sentiment polarities of the review titles. So we hypothesize the following:

Hypothesis H1b: The length of a review title has a stronger positive effect on its perceived helpfulness for Indian users than American users.

Hypothesis $\mathrm{H} 2 \mathrm{~b}$ : The positive sentiment of a review title has a stronger positive effect on the perceived helpfulness for Indian users than American users.

Hypothesis $\mathrm{H} 3 \mathrm{~b}$ : The negative sentiment of a review title has a stronger positive effect on its perceived helpfulness for Indian users than American users.

\section{Effect of review content characteristics on helpfulness}

Extant literature suggests that review content is an integral measure of the perceived helpfulness of online consumer reviews (Salehan and Kim 2016; Ismagilova et al. 2019). OCRs primarily consist of textual comments, ratings, and emoticons (Park and Kim 2008), which can signal the assessments of the positive or negative qualities of a product by the reviewers (Salehan and Kim 2016; Ghasemaghaei et al. 2018; Fresneda and Gefen (2019); Ismagilova et al. 2020). For instance, words that indicate joy, satisfaction, trust, and peace, such as love, nice, sweet, happy, and good, generate positive sentiments (Liu 2012; Nassirtoussi et al. 2014). Whereas words such as ugly, bad, poor, sick, and nasty evoke sadness, anger, anxiety, and frustration for the low quality of products or unfulfilled requirements and therefore generate negative sentiments (Yin et al. 2014; Chua and Banerjee 2016; Malik and Hussain 2017; Ghasemaghaei et al. 2018). Thus, we propose the following hypotheses:

Hypothesis H4a: The positive sentiment of a review-text has a positive effect on its perceived helpfulness. Hypothesis H5a: The negative sentiment of a review-text has a positive effect on its perceived helpfulness.
Next, a review-text's higher analytical content reflects more structured and logical thinking instead of an unstructured pattern. Greater use of nouns, articles, and prepositions indicates a highly analytical review-text. In contrast, fewer pronouns, auxiliary verbs (such as- am, will, have), and common adverbs (such as- very, really) are associated with low (or unstructured) analytical thinking (Pennebaker et al. 2015). Thus, we hypothesize:

Hypothesis H6a: The analytical nature of a review-text has a positive effect on its perceived helpfulness.

An OCR's readability is the level of comprehension required to understand it and make an informed decision while using it (Ghose and Ipeirotis 2010; Krishnamoorthy 2015; Agnihotri and Bhattacharya 2016; Chua and Banerjee 2016; Fresneda and Gefen 2019). Specifically, the readability of an OCR is directly related to its clarity (Biswas et al. 2020). The readability of a review-text may also improve the purchasing decisions related to the product (Korfiatis et al. 2012). Therefore, using a highly readable and lucid review-text leads to enhanced outcomes for e-marketplaces, such as higher purchase intentions (Jiménez and Mendoza 2013) or helpful reviews (Agnihotri and Bhattacharya 2016). Additionally, the readability of a review is affected by inaccurate punctuations and incorrect grammatical phrases that may negatively affect the primary positive effect of readability on the perceived helpfulness (Ghose and Ipeirotis 2010; Ahmad and Laroche 2015). Thus, a moderating effect of the grammatical completeness toward the review-readability is expected for online consumer reviews. Therefore, we hypothesize that:

Hypothesis H7a: The grammatical completeness of a review-text positively moderates the effect of review readability on perceived helpfulness

While reading an OCR, prospective users may perceive a vivid feeling of consuming the product leading to an indirect consumption experience (Racherla and Friske 2012; Liu and Park 2015). This effect is further enhanced by establishing a higher social connection between the reader and the OCR. These social processes may be indicated by family-related words (such as home, dad, brother); friends related words (such as buddy, friend, neighbor); female-related words (such as girl, her, mom); and male-related words (such as boy, his, dad). Consequently, a review's social nature combines the essence of social connections established between a reviewer and a reader. Thus, we hypothesize:

Hypothesis H8a: A review-text that displays higher social connect has a positive effect on its perceived helpfulness. 


\section{Cultural influence on review content characteristics}

Extant studies show that masculine cultures are more of doing and acquiring type than thinking and observing (Newman and Nollen 1996; Cyr et al. 2017; El Ghoul and Zheng 2016). For instance, OCRs in highly masculine cultures are more concerned with doing activities such as buying and acquiring (information about a product) rather than just observing. In the advertising domain, Wang (2008) has shown that females have a more favourable attitude towards helping when exposed to negative sentiments than positive and rational ones. Often users from highly feminine cultures emphasize thinking and observing actions (Newman and Nollen 1996; El Ghoul and Zheng 2016). Therefore, the sentimental content of an OCR would be more appealing to them than to the users from highly masculine cultures (Gunkel et al. 2014; Miao et al. 2018). Hence, we posit that Indian users who are high on femininity than American users shall emphasize the reviewtext sentiments to perceive an OCR's helpfulness. Therefore, we hypothesize:

Hypothesis H4b: The positive sentiment of a review-text has a stronger positive effect on its perceived helpfulness for Indian users than American users.

Hypothesis H5b: The negative sentiment of a review-text has a stronger positive effect on its perceived helpfulness for Indian users than American users.

Femininity relates to emotional, caring, and nurturing values (Johnston and Johal 1999; Gunkel et al. 2014, 2016; Miao et al. 2018) that connect to OCRs with high analytical content. Newman and Nollen (1996) argued that countries with a high masculine culture impart more emphasis on achievements or outcomes and abhor failures. Whereas, in countries with high feminine culture, the emphasis is on affiliation and quality of life, which is deemed a sign of success, and so users from these countries do not consider failure to be a significant consequence. Therefore, in this study, we claim that strong feminine cultures would emphasize the analytical nature of an OCR as they need not accentuate the absolute outcome or on the performance of the associated product but rather on the essentials of its usage. So, national cultures that are high on femininity (or low on masculinity) will convey more importance to the analytical content of an OCR, and therefore, prospective users will evaluate it to be more helpful. Thus, we posit that Indian users being high on femininity than American users, will emphasize a review's analytical content. So, we hypothesize:

Hypothesis H6b: The analytical nature of a review-text has a stronger positive effect on its perceived helpfulness for Indian users than American users.
Users from high uncertainty-avoidance countries tend to have a low tolerance for uncertain and ambiguous situations (Singh 2002; Ganguly et al. 2010; Tang 2017; Yu et al. 2018). These customers exhibit a higher perceived risk from using online information sources without appropriate credibility (Ganguly et al. 2010; Tang 2017; Yu et al. 2018; Rosillo-Díaz et al. 2019). Sometimes, these customers tend to be risk-averse to incoherent information and devise a well-structured environment instead (Nath and Murthy 2004; Shiu et al. 2015; Lu et al. 2018). Therefore, incorrect punctuations and incomplete sentences must be avoided while exploring the effects of readability on the perceived helpfulness (Ghose and Ipeirotis 2010). Further, in an ecommerce platform, prospective customers are apprehensive about the user experience, the credibility of displayed products, and associated reviews before the actual purchase. In this way, the search process creates an ambiguous scenario, which worsens for high uncertainty-avoidance users. However, the ease of reading a lucid review-text reduces this uncertainty. As American users are high on uncertainty-avoidance, we argue that they would emphasize readability to perceive an online consumer review to be helpful. Thus, we hypothesize:

Hypothesis H7b: The effect of readability moderated by grammatical completeness has a stronger positive effect on its perceived helpfulness for American users than Indian users.

Next, we discuss the effect of Hofstede's collectivism-individualism dimension on the perceived helpfulness of online consumer reviews. Collectivists prefer civic relationships and interpersonal harmony over personal choices, such as formal communitybuilding and social networks, to disseminate ideas (Singh 2002; Tang 2017; Rosillo-Díaz et al. 2019). In this way, they can simulate the presence of a physical marketplace in an online platform. For the same reason, prospective e-commerce buyers who are high on collectivism assign higher importance to what their friends, parents, and colleagues tell them than the users from individualistic societies (Tan et al. 2007; Tang 2017; Hong et al. 2016; Rosillo-Díaz et al. 2019). Often, the OCRs consist of family-related words (such as home, dad, brother), friendship-related words (such as buddy, friend, neighbor), all of which may make the review more helpful to consumers from high collectivist societies. Because Indian users score higher on collectivism than American users, we hypothesize:

Hypothesis H8b: A review-text with higher social connect has a stronger positive effect on its perceived helpfulness for Indian users than American users. 


\section{Effect of star ratings on helpfulness}

In an e-commerce platform, products receive star ratings from previous users to indicate the overall opinion and purchasing experience. Extant studies have reported the effect of stars toward the perceived helpfulness of OCRs, often as peripheral cues (Baek et al. 2012); review valence (Ngo-Ye et al. 2017; Wang and Karimi 2019; Ismagilova et al. 2019); review extremity (Mudambi and Schuff 2010) and ratings (Ghose and Ipeirotis 2010; Chua and Banerjee 2015; Chua and Banerjee 2016). Ratings are often shown in five-point star recommendations on the e-marketplace and influence an OCR's helpfulness (You et al. 2012; Lee and Choeh 2016; Ngo-Ye et al. 2017). Therefore,

Hypothesis H9a: The number of star ratings received by a review positively affects its perceived helpfulness.

\section{Cultural influence on star ratings}

According to Hofstede (1984), individuals from a high power distance culture can easily accept the unequal distribution of power. They also believe that other people in higher power positions can perform tasks that others with relatively lower power positions cannot perform (Tang 2017; Stamolampros et al. 2019). Therefore, e-commerce platforms used by individuals with high power distance cultures may emphasize those features related to power and prestige (Gao et al. 2018). Typically, the number of stars represents this embedded power, control, and psychological security among its readers. As the Indian users mostly hail from a culture that exhibits high power distance as compared to the American users, we hypothesize,

Hypothesis H9b: The number of star ratings received by a review has a stronger positive effect on its perceived helpfulness for Indian users than American users.

\section{Effect of temporal review characteristics on helpfulness}

Usually, potential customers on an e-commerce platform either focus on the future usage of the product or concentrate on its present performance, referred to as the time-orientation or temporal focus of an OCR. Therefore, users will perceive an online consumer review as helpful based on its available temporal features and facilitate purchase decisions ( $\mathrm{Hu}$ et al. 2008; Zhu et al. 2017; Stamolampros et al. 2019). Common words in the review-text that indicate a present temporal focus are today, is, and now, while words such as: may, will, and soon indicate the future temporal focus of the online consumer reviews. Thus we hypothesize that:

Hypothesis H10a: Present temporal focus of a review positively affects its perceived helpfulness.

Hypothesis H11a: Future temporal focus of a review positively affects its perceived helpfulness.

\section{Cultural influence on review temporal characteristics}

Long-term versus short-term orientation distinguishes societies that are focused on long-term goals, such as the Asian countries (India, China, Japan), from those who are more focused on immediate goals, such as North America (USA, Canada) and Europe (Germany, France) (Hofstede 1984; Steenkamp et al. 1999; Søndergaard 1994; Minkov and Hofstede 2012). Users who possess a short-term time orientation may relate to current events that are psychologically close. However, users with a long-term orientation may focus on events that are somewhat unpredictable and are psychologically distant (Zhu et al. 2017; Stamolampros et al. 2019). Therefore, the Indian customers prefer reviews that consist of descriptions about the product performance in the long run than in the present. American users, on the other hand, are happy to know what the product is currently doing. Thus we hypothesize that:

Hypothesis H10b: Present Focus has a stronger positive effect on the perceived helpfulness of a review for American users than Indian users.

Hypothesis H11b: Future Focus has a stronger positive effect on the perceived helpfulness of a review for Indian users than American users.

\section{Research methodology}

\section{Data used in our study}

This study analyzed online consumer reviews for multiple ranges of products that we collected from Amazon India (www.amazon.in) and Amazon USA (www.amazon.com). We chose the following products from Amazon India and Amazon USA: Amazon Echo Dot, Samsung Earbuds, JBL Headphones, Kaspersky Anti-virus, AmazonBasics battery, WD hard drive, HP printer cartridge, and AmazonBasics Classic Backpack. The reasons were: (a) Most of these products are manufactured and marketed by Amazon, so there are lesser chances of retail arbitrage effects. Therefore, prices remain uniform across sellers from different countries. (b) These products are a perfect mixture of "search" and "experience" (Nakayama et al. 2010). 
Next, we built a web-scraper in R specifically for this study and executed it to retrieve the recent reviews on Amazon India (14,129 reviews) and Amazon USA (13,811 reviews) up to September 2020. The extracted reviews consisted of mixed data-types, including numbers, texts, and timestamps. We marked the count of helpful votes as the dependent variable during our analysis and measured the perceived helpfulness of the OCRs that the previous users had submitted. Next, based on our proposed framework (Fig. 2), we extracted each review's relevant features that contributed to its helpfulness using textmining and data analysis (Nassirtoussi et al. 2014; Akter and Wamba 2016). We categorized those features into three types: (i) review title-based attributes: length, positive sentiments, negative sentiments, (ii) review content-based attributes: positive sentiments, negative sentiments, analytical content, readability, grammatical completeness, and social content; and (iii) temporal attributes: present focus, future focus.

We conducted a text-analysis with the Linguistic Inquiry Word Count (LIWC) software (Pennebaker et al. 2015). LIWC performs text analysis based on its inbuilt dictionary consisting of almost 6400 words, word stems, and emoticons as a well-known and reliable psychometric tool. The validity of LIWC analysis has been confirmed in numerous studies to examine the predictors of review helpfulness (Ludwig et al. 2013; Pennebaker et al. 2015; Agnihotri and Bhattacharya 2016; Ngo-Ye et al. 2017; Malik and Hussain 2017; Guo and Zhou 2017; Wang and Karimi 2019). Table 3 presents a brief description of the variables used in our study. We present a detailed measurement for each variable in the Supplementary Table A11.

While computing the readability indices of the review-text, we adopt the Simple Measure of Gobbledygook (SMOG) Index to examine readability. However, to avoid the instances where incomplete sentences and incorrect punctuations influence the readability index, we introduced a dummy variable which takes values: 1 (when the review-text is complete and grammatically accurate, e.g., ending with “.”, “!”, ,’?”), else 0 (for incomplete sentences without appropriate punctuations, where neither of these punctuations is to be found). We introduced an interaction effect of this dummy variable with the SMOG Index, while the SMOG Index's extreme values were eliminated. Table A1 (supplementary) and Table A2 (supplementary) illustrate the descriptive statistics of the variables from Amazon India and Amazon USA, respectively. Further, we checked the pairwise correlation among the chosen predictor variables to check for multicollinearity conditions. Table A3 and Table A4 (supplementary) illustrate the pairwise correlation between Amazon India and Amazon USA variables, respectively. These results demonstrate that the chosen predictors are free from multicollinearity problems.

\section{Empirical modelling}

As the response variable (i.e., helpfulness vote) was countable, our natural choice for a predictive model was Poisson regression. However, while inspecting the response
Fig. 2 Proposed conceptual framework with associated variables

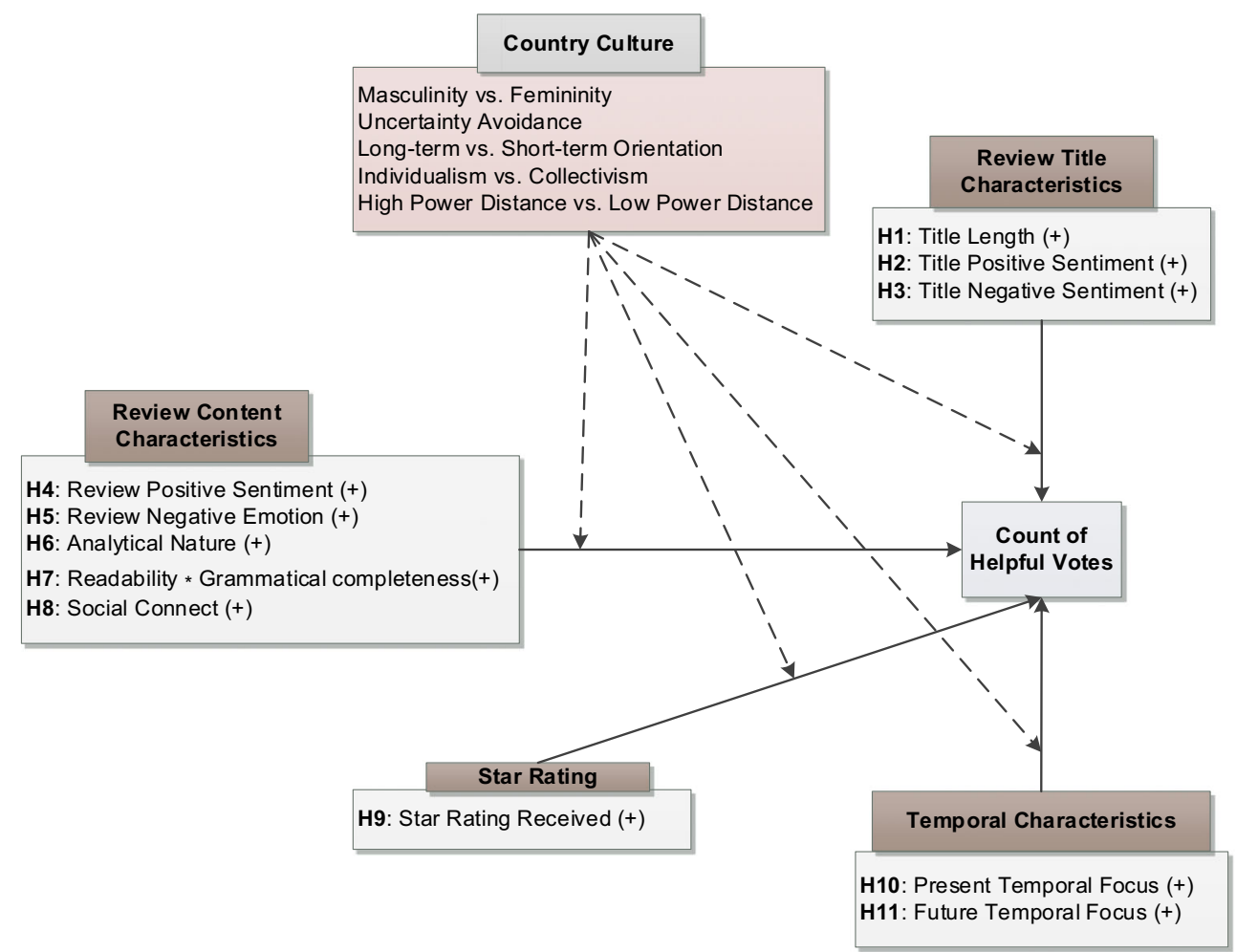


Table 3 Variables used to build our framework - brief descriptions, and literature sources

\begin{tabular}{|c|c|c|c|}
\hline S. no. & Variable & Brief Description & Literature source \\
\hline \multicolumn{4}{|c|}{ Review title } \\
\hline 1 & Title Length & Count of Words in the Review Title & Qazi et al. (2016); Salehan and Kim (2016) \\
\hline 2 & Title Positive Sentiment & Positive sentiments of Review Title & $\begin{array}{l}\text { Developed for this study from Liu (2012); } \\
\text { Salehan and Kim (2016) }\end{array}$ \\
\hline 3 & Title Negative Sentiment & Negative sentiments of Review Title & $\begin{array}{l}\text { Developed for this study from Liu (2012); } \\
\text { Salehan and Kim (2016) }\end{array}$ \\
\hline \multicolumn{4}{|c|}{ Review content } \\
\hline 4 & Review Positive Sentiment & Positive sentiments of Review Text & $\begin{array}{l}\text { Developed for this study from Liu (2012); } \\
\text { Salehan and Kim (2016); } \\
\text { Agnihotri and Bhattacharya (2016) }\end{array}$ \\
\hline 5 & Review Positive Sentiment & Negative sentiments of Review Text & $\begin{array}{l}\text { Developed for this study from Liu (2012); } \\
\text { Salehan and Kim (2016) } \\
\text { Agnihotri and Bhattacharya (2016); } \\
\text { Ghasemaghaei et al. (2018) }\end{array}$ \\
\hline 6 & Analytic & $\begin{array}{l}\text { Analytical and Logical Thinking of } \\
\text { Review Text }\end{array}$ & $\begin{array}{l}\text { Developed for this study from } \\
\text { Nassirtoussi et al. (2014) }\end{array}$ \\
\hline 7 & Readability & Measured by SMOG Index & $\begin{array}{l}\text { Ghose and Ipeirotis (2010); } \\
\text { Agnihotri and Bhattacharya (2016); } \\
\text { Malik and Hussain (2017); } \\
\text { Fresneda and Gefen (2019) }\end{array}$ \\
\hline 8 & Grammatical Completeness & $\begin{array}{l}\text { Absence of Incorrect Punctuations in } \\
\text { Review Text }\end{array}$ & Developed from Ghose and Ipeirotis (2010) \\
\hline 9 & Social & Socially Relevant Content in Review Text & Developed for this study \\
\hline \multicolumn{4}{|c|}{ Star rating } \\
\hline 10 & Stars & Star Ratings (out of 5) assigned to Review & $\begin{array}{l}\text { Mudambi and Schuff (2010); } \\
\text { Huang et al. (2015); Wang (2008); } \\
\text { Wang and Karimi (2019) }\end{array}$ \\
\hline 11 & Focuspresent & Time orientation of Review with "present focus" & Developed for this study \\
\hline 12 & Focusfuture & Time orientation of Review with "future focus" & Developed for this study \\
\hline 13 & Helpfulness & Count of Helpful Votes in a Review & $\begin{array}{l}\text { Mudambi and Schuff (2010); } \\
\text { Huang et al. (2015); Chua and Banerjee (2015); } \\
\text { Chua and Banerjee (2016); Filieri et al. (2018); } \\
\text { Wang et al. (2019); Wang and Karimi (2019) }\end{array}$ \\
\hline
\end{tabular}

variable, we noticed that users did not mark a significant number of reviews to be helpful, and so helpfulness votes were absent for those reviews: 12614 out of 14,129 OCRs for India and 11,182 out of 13,811 OCRs for the USA, respectively. This phenomenon is common among the OCR data and unnecessarily inflates the frequency of zero counts in the overall sample. Many existing scholars (Chua and Banerjee 2015; Salehan and Kim 2016; Agnihotri and Bhattacharya 2016; Malik and Hussain 2017; Barbro et al. 2020) have ignored reviews that have no associated helpfulness votes. However, such a treatment completely ignores (i) the count nature of the helpfulness votes, (ii) inflated count of non-voted reviews (zero counts), and (iii) drastically reduces the power of the final predictive model. Therefore, we propose to apply the zero-inflated Poisson (ZIP) regression, which can handle zero-inflated OCR data fluently and generate results with robust predictive powers (Lambert 1992). For the details on ZIP regression, please refer to Eqns. (A1)-(A2) (supplementary).
In addition to the count-data models mentioned above, we observed that the frequency of zero helpful votes was unusually high compared to other counts of helpful votes (refer Fig. 3), which leads to the problem of inflated counts and results in overdispersion. However, conditional mean-based regression may be sensitive to outliers in the response variable. We consider a quantile regression approach to model overdispersed count data (Fuzi et al. 2016; Ling 2019). For the details on quantile regression, please refer to Eq. (A3) (supplementary).

\section{Results and discussion}

To study the effect of the variables distinctly, we applied our proposed framework across four separate models in a stepwise manner where M1: predictors based on review-title; M2: predictors based on review-content; M3: predictors from M1, 
Fig. 3 a Density plot for the count of helpful votes demonstrating a zero-inflated behaviour (Amazon India). b Density plot for the count of helpful votes demonstrating a zero-inflated behaviour (Amazon USA)

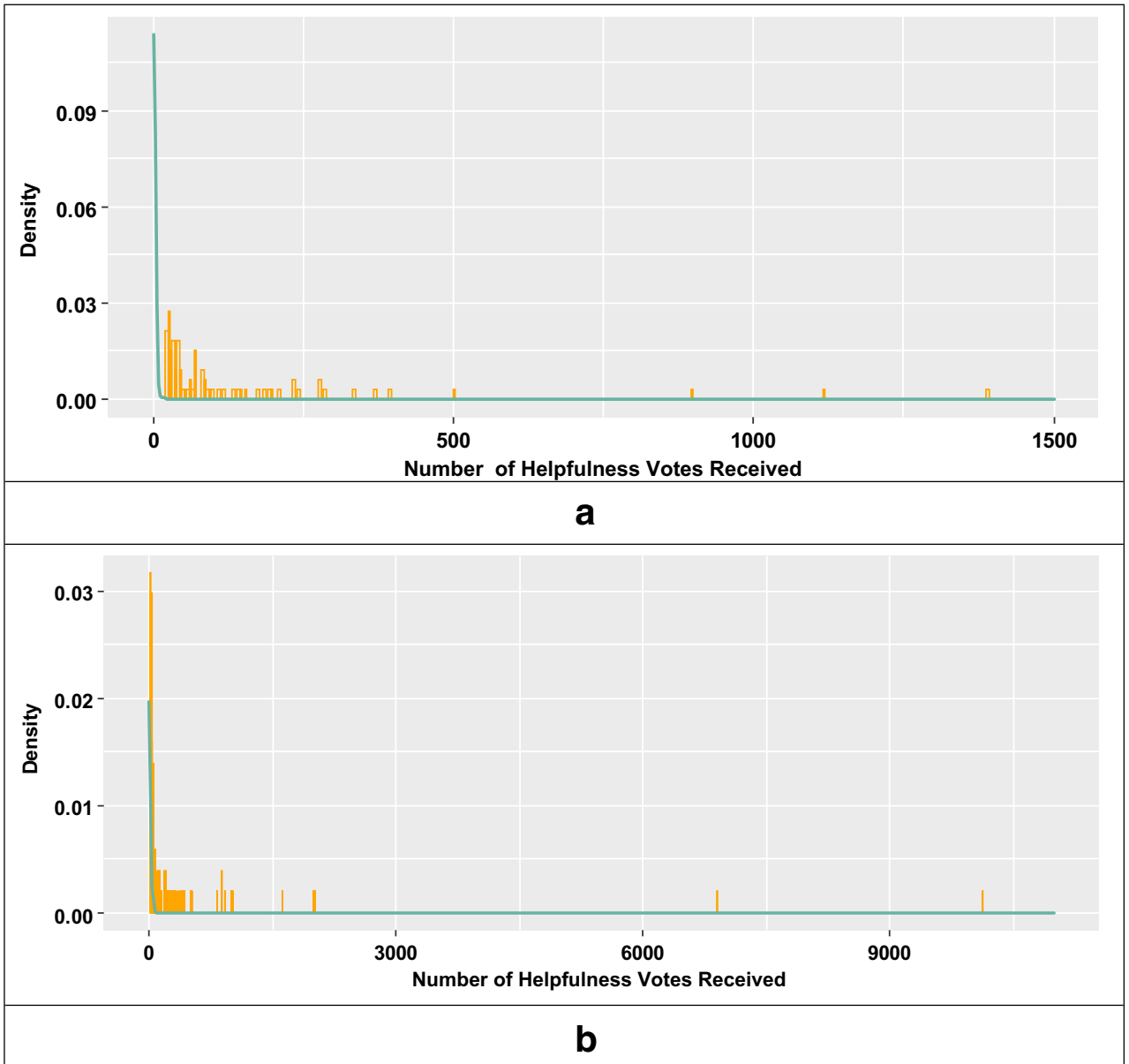

M2, and stars; and M4: the entire set of predictors from Table 3. Tables A5 and A6 (Supplementary) present the coefficient estimates for Amazon India and Amazon USA, respectively. Table A7 (Supplementary) presents the summary of hypotheses H1(a)-H11(a) for the main effects, while Table A8 (Supplementary) presents the summary of hypotheses H1(b) - H11(b) for the effects of culture.

Results for the main effects (Table A7) indicate that among the review-title characteristics, title length (India: $\beta=0.187$, USA: $\beta=0.009$ ), positive sentiments (India: $\beta=0.005$, USA: $\beta=0.001$ ) and negative sentiments (India: $\beta=0.014$, USA: $\beta=0.016$ ) are statistically significant and positive for both Amazon India and Amazon USA. The coefficient estimates support $\mathrm{H} 1 \mathrm{a}, \mathrm{H} 2 \mathrm{a}$, and $\mathrm{H} 3 \mathrm{a}$ for both Amazon India and Amazon USA. These findings are similar to those reported by Qazi et al. (2016); González-Rodríguez et al. (2016); Salehan and Kim (2016). Next, we found that Indian users who are low on masculinity had imparted higher importance to the review-title's sentiment polarities than American users. The title length had a higher coefficient estimate for Amazon India reviews than those from Amazon USA, thus supporting H1b. We also found that the e-commerce users for Amazon India imparted a higher significance to the positive sentiments and a relatively lower significance to the negative sentiments embedded in the review-title than the e-commerce users of Amazon USA. Thus, our empirical model provided evidence to support H2b, while H3b was not supported (Supplementary Table A8). Similar findings were reported by Stamolampros et al. (2019) while examining the service-quality perceptions for Airlines.

Next, among the review-content characteristics, social context (India: $\beta=0.039$, USA: $\beta=0.029$ ) emerged statistically significant for Amazon India and Amazon USA. Whereas, review readability moderated by grammatical completeness (India: $\beta=-0.073$, USA: $\beta=-0.038$ ) emerged statistically significant but with negative signs for both Amazon India and the USA. This finding is opposite to the relationship expected between review helpfulness and readability of an OCR. Previous studies for review-polarity (Baek et al. 2012; Ghasemaghaei et al. 2018; Fresneda and Gefen 2019; Ismagilova et al. 2020); semantics (Guo and Zhou 2017); spellings and grammatical completeness (Ghose and Ipeirotis 2010; Krishnamoorthy 2015) and review-readability (Malik and Hussain 2017; Fresneda and Gefen 2019) reported a positive coefficient. For the reviews from Amazon India, both positive sentiments (India: 
$\beta=-0.022$ ) and negative sentiments (India: $\beta=-0.021$ ), as well as analytical thinking (India: $\beta=-0.001$ ) of a review, had a negative effect on its perceived helpfulness. Therefore, $\mathrm{H} 4 \mathrm{a}, \mathrm{H} 5 \mathrm{a}, \mathrm{H} 6 \mathrm{a}$, and H7a were not supported, whereas H8a was supported for Amazon India.

For Amazon USA, both positive sentiments (USA: $\beta=-0.044$ ) and negative sentiments (USA: $\beta=-0.294$ ) of a review imparted a strong negative effect on its perceived helpfulness. So, no support was found for $\mathrm{H} 4 \mathrm{a}, \mathrm{H} 5 \mathrm{a}$, and $\mathrm{H} 7 \mathrm{a}$ for the e-commerce users of Amazon USA. Additionally, analytical thinking (USA: $\beta=0.004$ ) of the review conveyed a strong positive effect on its perceived helpfulness for Amazon USA. Therefore, hypotheses H6a and H8a were all supported by Amazon USA (Table A7). Next, comparing the coefficient estimates for both positive sentiments and negative sentiments, we observed that the reviews posted on Amazon USA had a stronger negative influence on the perceived helpfulness than those on Amazon India. Similar findings were reported for national culture's effect on UGC (Tang 2017) and airline service-quality (Stamolampros et al. 2019). Therefore, both H4b and H5b were supported. Whereas, the analytical content of the reviews for Amazon USA had a stronger positive influence on the perceived helpfulness than those for Amazon India, which is counterintuitive. Again, the review-text readability (moderated by the grammatical completeness) of the reviews posted on Amazon India posed a stronger negative influence on the perceived helpfulness than those for Amazon USA. Similar cross-cultural differences were attributed to high uncertainty-avoidance while selling luxury goods with quality labels (Yu et al. 2018) and perceived risks in e-commerce (Rosillo-Díaz et al. 2019). Nevertheless, if we examine the difference in estimates (India: $\beta=-0.073$, USA: $\beta=-0.038$ ), Indian users had a weaker positive influence than the American users. Therefore, H6b lacks support, while H7b is partially supported by our study (Supplementary Table A8).

Extant studies suggest that national cultures with high femininity convey more importance to an OCR's analytical content and perceive it more helpful (Tang 2017; Miao et al. 2018). However, from our study, we find counterintuitive results that do not support the prevailing cultural beliefs. Feminine cultures are more of thinking and observing type, rather than masculine cultures who are more of doing and acquiring type (Newman and Nollen 1996; Cyr et al. 2017; El Ghoul and Zheng 2016). Further, femininity relates to strong emotional values, such as caring and nurturing (Hofstede 1984; Johnston and Johal 1999; Gunkel et al. 2014; Gunkel et al. 2016). However, the results of our study did not support H8b. It could be because societies might be undergoing a change, and femininity could be rising (Ganguly et al. 2010). Hence, from our study, we find evidence to support H4b, H5b, H7b, and H8b but not for H6b (Table A8).
Next, we examined the effects of star-ratings on the perceived helpfulness of a review. Typically, stars enclose the overall opinion of preceding reviewers about a product or service on the e-commerce platform. Studies show that users from a society with high power distance can easily accept the unequal distribution of power and control (Hofstede 1984; Cyr 2008; Tang 2017; Stamolampros et al. 2019). The power distance scores for India and the USA are 77 and 40, respectively. ${ }^{7}$ The number of stars for a review represents these embedded power, control, and psychological security. Therefore, India's e-commerce users associate trustworthiness and status with reviews that received high star ratings and voted them to be more helpful than e-commerce users from the USA. The main results (India: $\beta=0.086$; USA: $\beta=$ 0.272 ) from our study show that reviews from both Amazon USA and Amazon India support H9a. Similar findings were reported for review valence and stars (Baek et al. 2012; Wang and Karimi 2019; Ismagilova et al. 2020). In terms of cultural differences, we find that e-commerce users belonging to the USA (where the societal structure has a low power distance) exercise a stronger positive impact of star ratings on the perceived helpfulness than e-commerce users from India, providing evidence against $\mathrm{H} 9 \mathrm{~b}$. A possible reason could be the categories of products that we analyzed: Amazon Echo, Samsung Earbuds, JBL Headphones, Kaspersky anti-virus, AmazonBasics battery, WD hard drive, HP printer cartridge, and AmazonBasics Classic Backpack. In India, these are purchased mostly by urban consumers, while the power distance in Indian societies is more prominent in the rural areas. A few rural consumers had possibly purchased these products, limiting the possibility of strong power distance traits.

Next, we examined the effect of the temporal focus of a review on its perceived helpfulness. Our results show that present temporal focus (India: $\beta=0.021$; USA: $\beta=-0.041$ ), as well as future temporal focus (India: $\beta=0.051$; USA: $\beta=$ -0.076 ), positively affect the perceived helpfulness of a review for the users of Amazon India while wielding a strong negative influence for the users of Amazon USA. Thus, we found strong support for H10a and H11a for India's ecommerce users, but not for USA (Table A7). These findings were unique for our study and have not been reported previously in the extant literature. Users who are high on short-term orientation typically think about current events that are psychologically close, while future-oriented people focus on unpredictable activities and are distant in the temporal dimension (Minkov and Hofstede 2012). Therefore, users from societies focused on long-term goals, such as those in India, may prefer reviews that focus on the future benefits of a product rather than its current usefulness (Zhu et al. 2017; Stamolampros

\footnotetext{
${ }^{7}$ Hofstede's Country Comparison: https://www.hofstede-insights.com/ country-comparison/india,the-usa/
} 
et al. 2019). On the contrary, societies that are dedicated to immediate goals in life, such as e-commerce users from the USA, may be satisfied with the current usage of the product only and search for short-term cues while reading product reviews. Therefore, our results support $\mathrm{H} 11 \mathrm{~b}$, while $\mathrm{H} 10 \mathrm{~b}$ is not supported (Table A8, supplementary).

\section{Discussion on the results from quantile regression}

We get some deeper insights into the effects of the explanatory variables for each quantile (Supplementary Table A9 and Table A10) for the entire distribution of the count of helpful votes. We found that the positive impact of title length (measured by word count) was significant for all levels of helpful reviews ( $p<0.001$ for Q1, Q2, .., Q8, and Q9) among customers from India, with relatively stronger effects for higher levels of the count of helpful votes (Fig. A1-a). Whereas, it was significant for only the higher levels of the count of helpful votes ( $p<0.1$ for Q7, Q8, and Q9) among customers from the USA, with relatively stronger effects for higher levels of the count of helpful votes (Fig. A2-a). The difference in the effects is possibly due to varying cross-cultural effects across e-commerce users from India and the USA and shows a stronger positive effect for Indian users than American users. Next, we found that the effect of readability of the review-text moderated by the grammatical completeness was significant for all levels of the count of helpful votes ( $p<0.001$ for Q1, Q2, .., Q8, and Q9) among customers from India, with relatively stronger negative effects for higher levels of the count of helpful votes (Q8 and Q9) (Fig. A1-b). Whereas, it was significant for all levels of the count of helpful votes ( $p<0.05$ for Q1, Q2, $\mathrm{Q} 3 ; p<0.001$ for $\mathrm{Q} 4, \mathrm{Q} 5, \ldots, \mathrm{Q} 9)$ among customers from the USA (Fig. A2-b). The difference in the effects shows a hypothesized weaker positive effect for Indian users than American users. Then, we found that the star rating was significant for all levels of the count of helpful votes among customers from India $(p<0.001$ for Q1, Q2, .., Q8, and Q9) (Fig. A1-c) and the USA $(p<0.001$ for Q1, Q2, .., $\mathrm{Q} 7 ; p<0.05$ for Q8 and Q9) (Fig. A2-c). When compared, the effects reveal a hypothesized stronger positive effect for Indian users than American users.

\section{Contributions to theory and practice}

\section{Theoretical contributions}

Our study has several theoretical contributions, mainly towards the social presence theory, self-efficacy theory, and Hofstede's Cultural framework in the context of online consumer reviews. First, our study extends the following two aspects of the social presence theory - (1) provides an improved measure of the perceived review helpfulness as a sense of social presence among the users of the e-commerce platform; (2) proposes innovative features such as the analytical content, readability, grammatical completeness, and social content that help to overcome the challenges of establishing interpersonal relationships in a virtual space.

Second, our study contributes to the following two aspects of the self-efficacy theory - (1) increases the self-efficacy of the potential buyers through a revised and more robust analytical lens to observe the "voted" as well as "non-voted" online consumer reviews, leading to a positive outcome (such as the product selection and subsequent purchase); (2) reduces the perceived complexity and knowledge barriers suffered by the potential buyers by apprising them through a set of novel review features.

Third, our study will help scholars searching for theories and frameworks to understand the cultural adaptation of online consumer reviews. There have been etic and emic approaches to analyze the cultural influences on a business in the past. The etic approach focuses on the universal aspects of culture, which is generalizable and can be used for crosscultural comparisons. On the other hand, the emic approach focuses on culture-specific issues, such as symbols that provide us with a detailed understanding of cultural and ethnic specifics. This study follows the etic approach, as our objective is to compare and contrast the factors that contribute to online consumer reviews and their perceived helpfulness across countries.

Fourth, our study contributes to the following two aspects of Hofstede's culture framework - (1) identify significant differences in the effect of the antecedents of helpfulness across different cultures; (2) explains these differences with the help of the five cultural dimensions of Hofstede. These differences were further elucidated with the help of the review attributes.

Fifth, we proposed a novel Zero-inflated Poisson regression to explain perceived review helpfulness with its predictors. We observed that the incidence of non-voted reviews was very high, leading to overdispersion effects addressed inadequately in previous studies. Sometimes, the review observations with zero votes were omitted during analysis. This practice might cause information asymmetry because ignoring those reviews did not indicate that a potential buyer has not read them. Hence, the Zero-inflated Poisson regression is a more suitable choice, given the current state of helpfulness votes.

\section{Managerial contributions}

Our study is also essential for managers due to the following reasons. First, it proposes a novel framework to forecast successful helpfulness votes (for both voted and non-voted reviews) in e-commerce platforms using an ensemble of predictors in the context of country-culture. This finding, we believe, would help the reviewers and critics to take improvement 
measures for highlighting various characteristics while writing reviews for products sold on the e-commerce platform.

Second, we identified a set of novel features, some of which were "explicit," such as the title length and star ratings, while others were "implicit features," such as title sentiments, review sentiments, review readability, analytical, social content, and time orientation. Our study demonstrated that most of these features profoundly affected the consumer's future purchasing decisions by perceiving the review as "helpful."

Third, online marketplaces cater to the needs of customers across different nationalities. Our study has shown that most of the factors contributing to perceived helpfulness can vary across country-cultures. Thus, business managers in e-commerce companies need to train critics and review-writers with the unique style necessary across e-commerce platforms in different countries (Nakayama and Wan 2019a, b). For instance, an Indian customer who possesses long-term time-orientation will look for a product's future usability. In contrast, an American customer who possesses short-term time-orientation will be satisfied with its current performance. Therefore the OCR to appeal to an Indian consumer significantly varies from an OCR submitted and read by American users.

Fourth, in the context of the recent coronavirus pandemic, it has become crucial for particular categories of businesses, especially the delivery of cooked food, groceries, vegetables, medicines, and other necessities to be purchased via ecommerce channels rather than from the physical stores ${ }^{8,9}$ Additionally, such unprecedented changes in the market conditions will push more small businesses and incumbent brickand-mortar stores worldwide to make their presence online and make their products available to a broader spectrum of customers. In such cases, prospective consumers will access reviews with a higher significance than before, leading to more complex e-commerce platforms (Alt and Zimmermann 2019).

Fifth, the influence of cultural dimensions on the determinants of online consumer reviews has much to do with the redesign of review systems and the redesign of e-commerce platforms (Stamolampros et al. 2019). For instance, customers from countries with high power distance such as India and the United Arab Emirates ${ }^{10}$ will relate product-details such as celebrity endorsement, seller's prestige, and the number of stars to its status, in contrast to users from countries with relatively low power distance such as USA and Germany.

\footnotetext{
${ }^{8}$ New York Times, Americans Keep Clicking to Buy, Minting New Online Shopping Winners: https://www.nytimes.com/interactive/2020/05/13/ technology/online-shopping-buying-sales-coronavirus.html

${ }^{9}$ Washington Post, Amazon sales soar as coronavirus-worried consumers shop from home, but costs rise: https://www. Washingtonpost.Com/ Technology/2020/04/30/Amazon-Earnings-Coronavirus/

${ }^{10}$ Hofstede Country Comparison: https://www.hofstede-insights.com/ country-comparison/germany, india,the-united-arab-emirates, the-usa/
}

Amazon India has recently launched "Global Stores" to help Indian sellers and small businesses sell their merchandise across marketplaces in the USA, Europe, and other countries. ${ }^{11}$ Therefore, it will be necessary for global buyers to adjust for the features (i.e., title length, stars, sentiments) of online consumer reviews and the helpfulness votes for crosscultural effects (Nakayama and Wan 2019a, b).

\section{Conclusion and future scope}

Our study offers actionable insights that involve identifying significant factors that contribute to the perceived helpfulness of online consumer reviews. In particular, we identified qualitative (such as readability, grammatical correctness, sentiments, social content, temporal focus) and quantitative (such as title length, star ratings) of UGCs on e-commerce platforms that affect the perceived helpfulness. We also identified how country culture influenced the relationship (Nakayama and Wan 2018, b; Stamolampros et al. 2019; Barbro et al. 2020) between the predictors of perceived helpfulness. To our knowledge, this is the first study that successfully applied Hofstede's framework to examine the cross-cultural effects in the context of OCRs and identifies their moderating effect on the perceived helpfulness (for both voted and non-voted reviews).

Specifically, this study identified the importance of several review title-based, review content-based, and review timeorientation based variables to explain the perceived helpfulness. Each review title-based attribute was significant for both India and the USA. Among review content-based attributes, positive sentiments, negative sentiments, and readability (moderated by grammatical completeness) were not significant, while social content was significant for India and the USA. Finally, variables based on time-orientation were significant for India but not for the USA. Throughout the study, we applied advanced text-mining techniques to extract unstructured data (Akter and Wamba 2016). These techniques may help develop market intelligence and product-based analytical knowledge that can be useful for sellers and manufacturers (Alt and Zimmermann 2019).

Despite these significant insights, our study has a few limitations. First, we applied the five dimensions proposed by Hofstede, namely power distance, masculinity-femininity, uncertainty avoidance, collectivism-individualism, and longterm vs. short-term orientation (Hofstede 1984; Hofstede et al. 1991). Future research could incorporate different cultural dimensions, such as high vs. low context (Hall and Hall 1989; Barbro et al. 2020), to extend this study. Second,

\footnotetext{
${ }^{11}$ Amazon Global Selling: https://services.amazon.in/services/amazonglobal-selling/benefits.html?ld=ASINAGSSEM_AGS_Branded_Export_ broad_main\&ld=ASINAGSSEM_519X_b_c_463606554-̄443
} 
throughout this study, we assumed that the country-level culture would act as a surrogate measure of individual cultural values. However, future research could apply other to identify online consumers' cultural values methods (Yoo et al. 2011). Finally, to offer additional objective evidence on our proposed framework's generalizability, future studies could involve using multiple product categories across different countries.

Supplementary Information The online version contains supplementary material available at https://doi.org/10.1007/s12525-020-00452-1.

Acknowledgements The authors gratefully acknowledge the valuable contributions from the Editors-in-Chief of this journal Hans-Dieter Zimmermann and Rainer Alt during the development of this paper. The authors also acknowledge the inputs received from the Guest Editors of the Special Issue and the anonymous reviewers during the revision process.

\section{References}

Agarwal, J., \& Wu, T. (2018). E-commerce in emerging economies: A multi-theoretical and multilevel framework and global firm strategies. In Emerging Issues in Global Marketing (pp. 231-253). Cham: Springer. https://doi.org/10.1007/978-3-319-74129-1_9.

Agnihotri, A., \& Bhattacharya, S. (2016). Online review helpfulness: Role of qualitative factors. Psychology \& Marketing, 33(11), 1006-1017. https://doi.org/10.1002/mar.20934.

Ahmad, S., \& Laroche, M. (2015). How do expressed emotions affect the helpfulness of a product review? Evidence from reviews using latent semantic analysis. International Journal of Electronic Commerce, 20(1), 76-111. https://doi.org/10.1080/10864415.2016.1061471.

Akter, S., \& Wamba, S. F. (2016). Big data analytics in E-commerce: a systematic review and agenda for future research. Electronic Markets, 26(2), 173-194. https://doi.org/10.1007/s12525-0160219-0.

Alt, R., \& Zimmermann, H.-D. (2019). Electronic markets on platform competition. Electronic Markets, 29(2), 143-149. https://doi.org/10. 1007/s12525-019-00353-y .

Babić Rosario, A., Sotgiu, F., De Valck, K., \& Bijmolt, T. H. (2016). The effect of electronic word of mouth on sales: A meta-analytic review of platform, product, and metric factors. Journal of Marketing Research, 53(3), 297-318. https://doi.org/10.1509/jmr.14.0380.

Baek, H., Ahn, J., \& Choi, Y. (2012). Helpfulness of online consumer reviews: readers' objectives and review cues. International Journal of Electronic Commerce, 17(2), 99-126. https://doi.org/10.2753/ JEC1086-4415170204.

Bandura, A. (1977). Self-efficacy: Toward a unifying theory of behavioral change. Psychological Review, 84(2), 191-215. https://doi.org/ 10.1037/0033-295X.84.2.191.

Barbro, P. A., Mudambi, S. M., \& Schuff, D. (2020). Do country and culture influence online reviews? An analysis of a multinational retailer's country-specific sites. Journal of International Consumer Marketing, 32(1), 1-14. https://doi.org/10.1080/08961530.2019. 1635552.

Biswas, B., Sengupta, P., Chatterjee, D. (2020). Examining the determinants of the count of customer reviews in peer-to-peer home-sharing platforms using clustering and count regression techniques. Decision Support Systems, 135. https://doi.org/10.1016/j.dss.2020. 113324.
Cao, Q., Duan, W., \& Gan, Q. (2011). Exploring determinants of voting for the "helpfulness" of online user reviews: A text mining approach. Decision Support Systems, 50(2), 511-521. https://doi.org/ 10.1016/j.dss.2010.11.009.

Choi, Y. K., Miracle, G. E., \& Biocca, F. (2001). The effects of anthropomorphic agents on advertising effectiveness and the mediating role of presence. Journal of Interactive Advertising, 2(1), 1932. https://doi.org/10.1080/15252019.2001.10722055.

Chua, A. Y., \& Banerjee, S. (2015). Understanding review helpfulness as a function of reviewer reputation, review rating, and review depth. Journal of the Association for Information Science and Technology, 66(2), 354-362. https://doi.org/10.1002/asi.23180.

Chua, A. Y., \& Banerjee, S. (2016). Helpfulness of user-generated reviews as a function of review sentiment, product type and information quality. Computers in Human Behavior, 54, 547-554. https:// doi.org/10.1016/j.chb.2015.08.057.

Cyr, D. (2008). Modeling web site design across cultures: relationships to trust, satisfaction, and e-loyalty. Journal of Management Information Systems, 24(4), 47-72. https://doi.org/10.2753/ MIS0742-1222240402.

Cyr, D., Gefen, D., \& Walczuch, R. (2017). Exploring the relative impact of biological sex and masculinity-femininity values on information technology use. Behaviour \& Information Technology, 36(2), 178193. https://doi.org/10.1080/0144929X.2016.1212091.

Dash, S., \& Saji, K. B. (2008). The role of consumer self-efficacy and website social-presence in customers' adoption of B2C online shopping: an empirical study in the Indian context. Journal of International Consumer Marketing, 20(2), 33-48. https://doi.org/ 10.1300/J046v20n02_04.

El Ghoul, S., \& Zheng, X. (2016). Trade credit provision and national culture. Journal of Corporate Finance, 41, 475-501. https://doi.org/ 10.1016/j.jcorpfin.2016.07.002.

El-Said, O. A. (2020). Impact of online reviews on hotel booking intention: the moderating role of brand image, star category, and price. Tourism Management Perspectives, 33, 100604. https://doi.org/10. 1016/j.tmp.2019.100604.

Erkan, I., \& Evans, C. (2016). The influence of eWOM in social media on consumers' purchase intentions: An extended approach to information adoption. Computers in Human Behavior, 61, 47-55. https:// doi.org/10.1016/j.chb.2016.03.003.

Filieri, R., Raguseo, E., \& Vitari, C. (2018). When are extreme ratings more helpful? Empirical evidence on the moderating effects of review characteristics and product type. Computers in Human Behavior, 88, 134-142. https://doi.org/10.1016/j.chb.2018.05.042.

Fishbein, M., \& Ajzen, I. (1975). Belief, attitude, intention, and behavior: Behavior: An introduction to theory and research. Reading: Addison-Wesley.

Flanagin, A. J., \& Metzger, M. J. (2013). Trusting expert-versus usergenerated ratings online: the role of information volume, valence, and consumer characteristics. Computers in Human Behavior, 29(4), 1626-1634. https://doi.org/10.1016/j.chb.2013.02.001.

Forman, C., Ghose, A., \& Wiesenfeld, B. (2008). Examining the relationship between reviews and sales: The role of reviewer identity disclosure in electronic markets. Information Systems Research, 19(3), 291-313. https://doi.org/10.1287/isre.1080.0193.

Fresneda, J. E., \& Gefen, D. (2019). A semantic measure of online review helpfulness and the importance of message entropy. Decision Support Systems, 125, 113117. https://doi.org/10.1016/j.dss.2019. 113117.

Friedrich, T., Schlauderer, S., \& Overhage, S. (2019). Some things are just better rich: how social commerce feature richness affects consumers' buying intention via social factors. Electronic Markets, $1-$ 22. https://doi.org/10.1007/s12525-019-00374-7.

Fu, D., Hong, Y., Wang, K., \& Fan, W. (2017). Effects of membership tier on user content generation behaviors: Evidence from online 
reviews. Electronic Commerce Research, 7, 1-27. https://doi.org/ 10.1007/s10660-017-9266-7.

Furner, C. P., Zinko, R., \& Zhu, Z. (2018). Examining the role of Mobile self-efficacy in the word-of-mouth/Mobile product reviews relationship. International Journal of E-Services and Mobile Applications, 10(4), 40-60. https://doi.org/10.4018/IJESMA.2018100103.

Fuzi, M. F. M., Jemain, A. A., \& Ismail, N. (2016). Bayesian quantile regression model for claim count data. Insurance: Mathematics and Economics, 6, 124-137. https://doi.org/10.1016/j.insmatheco.2015. 11.004.

Ganguly, B., Dash, S. B., Cyr, D., \& Head, M. (2010). The effects of website design on purchase intention in online shopping: the mediating role of trust and the moderating role of culture. International Journal of Electronic Business, 8(4-5), 302-330. https://doi.org/10. 1504/IJEB.2010.035289.

Gao, B., Li, X., Liu, S., \& Fang, D. (2018). How power distance affects online hotel ratings: the positive moderating roles of hotel chain and reviewers' travel experience. Tourism Management, 65, 176186. https://doi.org/10.1016/j.tourman.2017.10.007.

Ghasemaghaei, M., Eslami, S. P., Deal, K., \& Hassanein, K. (2018). Review length and sentiment as correlates of online reviews ratings. Internet Research, 28(3), 544-563. https://doi.org/10.1108/IntR-122016-0394.

Ghose, A., \& Ipeirotis, P. G. (2010). Estimating the helpfulness and economic impact of product reviews: mining text and reviewer characteristics. IEEE Transactions on Knowledge and Data Engineering, 23(10), 1498-1512. https://doi.org/10.1109/TKDE. 2010.188 .

González-Rodríguez, M. R., Martínez-Torres, R., \& Toral, S. (2016). Post-visit and pre-visit tourist destination image through eWOM sentiment analysis and perceived helpfulness. International Journal of Contemporary Hospitality Management, 28(11), 26092627. https://doi.org/10.1108/IJCHM-02-2015-0057.

Gunkel, M., Schlägel, C., \& Engle, R. L. (2014). Culture's influence on emotional intelligence: an empirical study of nine countries. Journal of International Management, 20, 256-274. https://doi.org/10.1016/ j.intman.2013.10.002.

Gunkel, M., Schlaegel, C., \& Taras, V. (2016). Cultural values, emotional intelligence, and conflict handling styles: a global study. Journal of World Business, 51, 568-585. https://doi.org/10.1016/j.jwb.2016. 02.001 .

Guo, B., \& Zhou, S. (2017). What makes population perception of review helpfulness: an information processing perspective. Electronic Commerce Research, 17(4), 585-608. https://doi.org/10.1007/ s10660-016-9234-7.

Hall, E. T., \& Hall, M. R. (1989). Understanding cultural differences. Intercultural Press.

Hernández-Ortega, B. (2018). Don't believe strangers: online consumer reviews and the role of social psychological distance. Information \& Management, 55(1), 31-50. https://doi.org/10.1016/j.im.2017.03. 007.

Hocevar, K. P., Flanagin, A. J., \& Metzger, M. J. (2014). Social media self-efficacy and information evaluation online. Computers in Human Behavior, 39, 254-262. https://doi.org/10.1016/j.chb.2014. 07.020

Hofstede, G. (1984). Culture's consequences: International differences in work-related values, volume 5 . Sage.

Hofstede, G. H., Hofstede, G. J., \& Minkov, M. (1991). Cultures and organizations: Software of the mind. New York: McGraw-Hill.

Hong, Y., Huang, N., Burtch, G., \& Li, C. (2016). Culture, conformity, and emotional suppression in online reviews. Journal of the Association for Information Systems, 17(11) Article 2.

Hong, H., Xu, D., Wang, G. A., \& Fan, W. (2017). Understanding the determinants of online review helpfulness: a meta-analytic investigation. Decision Support Systems, 102, 1-11. https://doi.org/10. 1016/j.dss.2017.06.007.
Hu, N., Liu, L., \& Zhang, J. J. (2008). Do online reviews affect product sales? The role of reviewer characteristics and temporal effects. Information Technology and Management, 9(3), 201-214. https:// doi.org/10.1007/s10799-008-0041-2.

Huang, A. H., Chen, K., Yen, D. C., \& Tran, T. P. (2015). A study of factors that contribute to online review helpfulness. Computers in Human Behavior, 48, 17-27. https://doi.org/10.1016/j.chb.2015.01. 010 .

Ismagilova, E., Slade, E. L., Rana, N. P., \& Dwivedi, Y. K. (2019). The effect of electronic word of mouth communications on intention to buy: a meta-analysis. Information Systems Frontiers., 1-24. https:// doi.org/10.1007/s10796-019-09924-y

Ismagilova, E., Dwivedi, Y. K., \& Slade, E. (2020). Perceived helpfulness of eWOM: Emotions, fairness and rationality. Journal of Retailing and Consumer Services, 53. https://doi.org/10.1016/j. jretconser.2019.02.002.

Jiménez, F. R., \& Mendoza, N. A. (2013). Too popular to ignore: The influence of online reviews on purchase intentions of search and experience products. Journal of Interactive Marketing, 27(3), 226235. https://doi.org/10.1016/j.intmar.2013.04.004.

Johnston, K., \& Johal, P. (1999). The internet as a "virtual cultural region": are extant cultural classification schemes appropriate? Internet Research, 9(3), 178-186. https://doi.org/10.1108/ 10662249910274566.

Kim, Y. H., \& Kim, D. J. (2005). A study of online transaction selfefficacy, consumer trust, and uncertainty reduction in electronic commerce transaction. In Proceedings of the 38th Annual Hawaii International Conference on System Sciences. 170c-170c. https:// doi.org/10.1109/HICSS.2005.52.

Kluckhohn, C., \& Kelly, W. H. (1945). The concept of culture. In R. Linton (Ed.), The science of man in the world crisis (pp. 78-106). New York: Columbia University Press.

Korfiatis, N., GarcíA-Bariocanal, E., \& SáNchez-Alonso, S. (2012). Evaluating content quality and helpfulness of online product reviews: the interplay of review helpfulness vs. review content. Electronic Commerce Research and Applications, 11(3), 205217. https://doi.org/10.1016/j.elerap.2011.10.003.

Krishnamoorthy, S. (2015). Linguistic features for review helpfulness prediction. Expert Systems with Applications, 42(7), 37513759. https://doi.org/10.1016/j.eswa.2014.12.044.

Kroeber, A. L., \& Kluckhohn, C. (1952). Culture: A critical review of concepts and definitions. Papers. Peabody Museum of Archaeology \& Ethnology, Harvard University.

Kuan, K. K., Hui, K.-L., Prasarnphanich, P., \& Lai, H.-Y. (2015). What makes a review voted? An empirical investigation of review voting in online review systems. Journal of the Association for Information Systems, 16(1), 48-71. https://doi.org/10.17705/1 jais.00386.

Lambert, D. (1992). Zero-inflated poisson regression, with an application to defects in manufacturing. Technometrics, 34(1), 1-14. https://doi. org/10.1080/00401706.1992.10485228.

Lee, S., \& Choeh, J. Y. (2016). The determinants of helpfulness of online reviews. Behaviour \& Information Technology, 35(10), 853863. https://doi.org/10.1080/0144929X.2016.1173099.

Lee, E. J., \& Shin, S. Y. (2014). When do consumers buy online product reviews? Effects of review quality, product type, and reviewer's photo. Computers in Human Behavior, 31, 356-366. https://doi. org/10.1016/j.chb.2013.10.050.

Li, J., \& Zhan, L. (2011). Online persuasion: How the written word drives WOM evidence from consumer-generated product reviews. Journal of Advertising Research, 51(1), 239-257. https://doi.org/10.2501/ JAR-51-1-239-257.

Li, M., Huang, L., Tan, C.-H., \& Wei, K.-K. (2013). Helpfulness of online product reviews as seen by consumers: source and content features. International Journal of Electronic Commerce, 17(4), 101-136. https://doi.org/10.2753/JEC1086-4415170404. 
Li, X., Wu, C., \& Mai, F. (2019a). The effect of online reviews on product sales: a joint sentiment-topic analysis. Information \& Management, 56(2), 172-184. https://doi.org/10.1016/j.im.2018.04.007.

Li, H., Fang, Y., Lim, K. H., \& Wang, Y. (2019b). Platform-based function repertoire, reputation, and sales performance of e-marketplace sellers. MIS Quarterly, 43(1), 207-236. https://doi.org/10.25300/ MISQ/2019/14201.

Li, K., Chen, Y., \& Zhang, L. (2020). Exploring the influence of online reviews and motivating factors on sales: a meta-analytic study and the moderating role of product category. Journal of Retailing and Consumer Services, 55, 102107. https://doi.org/10.1016/j. jretconser.2020.102107.

Ling, W. (2019). Quantile regression for zero-inflated outcomes. Ph.D. Thesis, Columbia University.

Liu, B. (2012). Sentiment analysis and opinion mining. Synthesis Lectures on Human Language Technologies, 5(1), 1-167. https:// doi.org/10.2200/S00416ED1V01Y201204HLT016.

Liu, Z., \& Park, S. (2015). What makes a useful online review? Implication for travel product websites. Tourism Management, 47, 140-151. https://doi.org/10.1016/j.tourman.2014.09.020.

Liu, Y., Feng, J., \& Liao, X. (2017). When online reviews meet sales volume information: is more or accurate information always better? Information Systems Research, 28, 723-743. https://doi.org/10. 1287/isre.2017.0715.

Liu, Z., Lei, S.-h., Guo, Y.-1., \& Zhou, Z.-a. (2020). The interaction effect of online review language style and product type on consumers' purchase intentions. Palgrave Communications, 6(1), 1-8. https:// doi.org/10.1057/s41599-020-0387-6.

Lo, A., \& Qu, H. (2015). A theoretical model of the impact of a bundle of determinants on tourists' visiting and shopping intentions: a case of mainland Chinese tourists. Journal of Retailing and Consumer Services, 22, 231-243. https://doi.org/10.1016/j.jretconser.2014.08.004.

Lu, B., Fan, W., \& Zhou, M. (2016). Social presence, trust, and social commerce purchase intention: an empirical research. Computers in Human Behavior, 56, 225-237. https://doi.org/10.1016/j.chb.2015. 11.057.

Lu, Q. S., Pattnaik, C., Xiao, J., \& Voola, R. (2018). Cross-national variation in consumers' retail channel selection in a multichannel environment: evidence from Asia-Pacific countries. Journal of Business Research, 86, 321-332. https://doi.org/10.1016/j.jbusres. 2017.09.027.

Ludwig, S., De Ruyter, K., Friedman, M., Brüggen, E. C., Wetzels, M., \& Pfann, G. (2013). More than words: the influence of affective content and linguistic style matches in online reviews on conversion rates. Journal of Marketing, 77(1), 87-103. https://doi.org/10. 2307/41714531.

Malik, M., \& Hussain, A. (2017). Helpfulness of product reviews as a function of discrete positive and negative emotions. Computers in Human Behavior, 73, 290-302. https://doi.org/10.1016/j.chb.2017. 03.053 .

McSweeney, B. (2002). Hofstede's model of national cultural differences and their consequences: a triumph of faith-a failure of analysis. Human Relations, 55(1), 89-118. https://doi.org/10.1177/ 0018726702551004.

Miao, C., Humphrey, R. H., \& Qian, S. (2018). A cross-cultural metaanalysis of how leader emotional intelligence influences subordinate task performance and organizational citizenship behavior. Journal of World Business, 53(4), 463-474. https://doi.org/10.1016/j.jwb. 2018.01.003.

Minkov, M., \& Hofstede, G. (2012). Hofstede's fifth dimension: new evidence from the world values survey. Journal of Cross-Cultural Psychology, 43(1), 3-14. https://doi.org/10.1177/ 0022022110388567.

Mudambi, S. M., \& Schuff, D. (2010). What makes a helpful review? A study of customer reviews on amazon.com. MIS Quarterly, 34(1), $185-200$.
Nakayama, M., \& Wan, Y. (2018). Is culture of origin associated with more expressions? An analysis of yelp reviews on Japanese restaurants. Tourism Management, 66. https://doi.org/10.1016/j.tourman. 2017.10.019.

Nakayama, M., \& Wan, Y. (2019a). Cross-cultural examination on content bias and helpfulness of online reviews : Sentiment balance at the aspect level for a subjective good. In HICSS 52, 6, 1154-1163. https://doi.org/10.24251/HICSS.2019.141.

Nakayama, M., \& Wan, Y. (2019b). The cultural impact on social commerce: a sentiment analysis on yelp ethnic restaurant reviews. Information and Management., 56, 271-279. https://doi.org/10. 1016/j.im.2018.09.004

Nakayama, M., Sutcliffe, N., \& Wan, Y. (2010). Has the web transformed experience goods into search goods? Electronic Markets, 20(3-4), 251-262. https://doi.org/10.1007/s12525-010-0041-z.

Nassirtoussi, A. K., Aghabozorgi, S., Wah, T. Y., \& Ngo, D. C. L. (2014). Text mining for market prediction: a systematic review. Expert Systems with Applications, 41(16), 7653-7670. https://doi.org/10. 1016/j.eswa.2014.06.009.

Nath, R., \& Murthy, N. (2004). A study of the relationship between internet diffusion and culture. Journal of International Information Management, 13(2), 5.

Newman, K. L., \& Nollen, S. D. (1996). Culture and congruence: the fit between management practices and national culture. Journal of International Business Studies, 27(4), 753-779. https://doi.org/10. 1057/palgrave.jibs.8490152.

Ngo-Ye, T. L., \& Sinha, A. P. (2014). The influence of reviewer engagement characteristics on online review helpfulness: a text regression model. Decision Support Systems, 61, 47-58. https://doi.org/10. 1016/j.dss.2014.01.011.

Ngo-Ye, T. L., Sinha, A. P., \& Sen, A. (2017). Predicting the helpfulness of online reviews using a scripts-enriched text regression model. Expert Systems with Applications, 71, 98-110. https://doi.org/10. 1016/j.eswa.2016.11.029.

Pai, P. F., \& Liu, C. H. (2018). Predicting vehicle sales by sentiment analysis of Twitter data and stock market values. IEEE Access, 6, 57655-57662. https://doi.org/10.1109/ACCESS.2018.2873730.

Park, D.-H., \& Kim, S. (2008). The effects of consumer knowledge on message processing of electronic word-of-mouth via online consumer reviews. Electronic Commerce Research and Applications, 7(4), 399-410. https://doi.org/10.1016/j.elerap.2007.12.001.

Park, I., Sarnikar, S., \& Cho, J. (2020). Disentangling the effects of efficacy-facilitating informational support on health resilience in online health communities based on phrase-level text analysis. Information \& Management, 103372. https://doi.org/10.1016/j.im. 2020.103372.

Pennebaker, J. W., Boyd, R. L., Jordan, K., \& Blackburn, K. (2015). The development and psychometric properties of LIWC2015. Technical Report.

Prince, N. R., Prince, J. B., \& Kabst, R. (2020). National culture and incentives: are incentive practices always good? Journal of World Business, 55(3), 101075. https://doi.org/10.1016/j.jwb.2020. 101075.

Qazi, A., Syed, K. B. S., Raj, R. G., Cambria, E., Tahir, M., \& Alghazzawi, D. (2016). A concept-level approach to the analysis of online review helpfulness. Computers in Human Behavior, 58, 75-81. https://doi.org/10.1016/j.chb.2015.12.028.

Racherla, P., \& Friske, W. (2012). Perceived usefulness of online consumer reviews: an exploratory investigation across three services categories. Electronic Commerce Research and Applications, 11(6), 548-559. https://doi.org/10.1016/j.elerap.2012.06.003.

Rosillo-Díaz, E., Blanco-Encomienda, F. J., \& Crespo-Almendros, E. (2019). A cross-cultural analysis of perceived product quality, perceived risk and purchase intention in e-commerce platforms. Journal of Enterprise Information Management, 33(1), 139160. https://doi.org/10.1108/JEIM-06-2019-0150. 
Salehan, M., \& Kim, D. J. (2016). Predicting the performance of online consumer reviews: a sentiment mining approach to big data analytics. Decision Support Systems, 81, 30-40. https://doi.org/10.1016/j. dss.2015.10.006.

Schindler, R. M., \& Bickart, B. (2012). Perceived helpfulness of online consumer reviews: the role of message content and style. Journal of Consumer Behaviour, 11, 234-243. https://doi.org/10.1002/cb. 1372.

Shiu, E., Walsh, G., Hassan, L. M., \& Parry, S. (2015). The direct and moderating influences of individual-level cultural values within web engagement: a multi-country analysis of a public information website. Journal of Business Research, 68(3), 534-541. https:// doi.org/10.1016/j.jbusres.2014.09.009.

Short, J., Williams, E., \& Christie, B. (1976). The social psychology of telecommunications. Wiley.

Singh, N. (2002). Analyzing cultural sensitivity of websites. Journal of Practical Global Business, 1(2), 32-53.

Søndergaard, M. (1994). Research note: Hofstede's consequences: a study of reviews, citations and replications. Organization Studies, 15(3), 447-456. https://doi.org/10.1177/017084069401500307.

Srivastava, S. C., \& Chandra, S. (2018). Social presence in virtual world collaboration: An uncertainty reduction perspective using a mixed methods approach. MIS Quarterly, 42(3), 779-804. https://doi.org/ 10.25300/MISQ/2018/11914.

Stamolampros, P., Korfiatis, N., Kourouthanassis, P., \& Symitsi, E. (2019). Flying to quality: cultural influences on online reviews. Journal of Travel Research, 58(3), 496-511. https://doi.org/10. 1177/0047287518764345

Steenkamp, J.-B. E., Ter Hofstede, F., \& Wedel, M. (1999). A crossnational investigation into the individual and national cultural antecedents of consumer innovativeness. Journal of Marketing, 63(2), 55-69. https://doi.org/10.1177/002224299906300204.

Sussman, S. W., \& Siegal, W. S. (2003). Informational influence in organizations: an integrated approach to knowledge adoption. Information Systems Research, 14(1), 47-65.

Tan, F. B., Yan, L. \& Urquhart, C. (2007). The effect of cultural differences on attitude, peer influence, external influence and self-efficacy in actual online shopping behavior. Journal of Information Science and Technology, 4(1), 3-26.

Tang, L. (2017). Mine your customers or mine your business: the moderating role of culture in online word-of-mouth reviews. Journal of International Marketing, 25(2), 88-110. https://doi.org/10.1509/ jim.16.0030.

Wang, A. (2008). Dimensions of corporate social responsibility and advertising practice. Corporate Reputation Review, 11(2), 155168. https://doi.org/10.1057/crr.2008.15.

Wang, F., \& Karimi, S. (2019). This product works well (for me): the impact of first-person singular pronouns on online review helpfulness. Journal of Business Research, 104, 283-294. https:// doi.org/10.1016/j.jbusres.2019.07.028.

Wang, Y., Wang, J., \& Yao, T. (2019). What makes a helpful online review? A meta-analysis of review characteristics. Electronic Commerce Research, 19(2), 257-284. https://doi.org/10.1007/ s10660-018-9310-2.

Wu, J., \& Liu, D. (2007). The effects of trust and enjoyment on intention to play online games. Journal of Electronic Commerce Research, $8(2)$.

$\mathrm{Xu}, \mathrm{Q}$. (2014). Should I trust him? The effects of reviewer profile characteristics on eWOM credibility. Computers in Human Behavior, 33, 136-144. https://doi.org/10.1016/j.chb.2014.01.027.

Yin, D., Bond, S., \& Zhang, H. (2014). Anxious or angry? Effects of discrete emotions on the perceived helpfulness of online reviews. MIS Quarterly, 38(2), 539-560.

Yoo, B., Donthu, N., \& Lenartowicz, T. (2011). Measuring Hofstede's five dimensions of cultural values at the individual level: Development and validation of CVSCALE. Journal of International Consumer Marketing, 23(3-4), 193-210. https://doi. org $/ 10.1080 / 08961530.2011 .578059$.

You, W., Xia, M., Liu, L., \& Liu, D. (2012). Customer knowledge discovery from online reviews. Electronic Markets, 22(3), 131142. https://doi.org/10.1007/s12525-012-0098-y.

Yu, S., Hudders, L., \& Cauberghe, V. (2018). Selling luxury products online: The effect of a quality label on risk perception, purchase intention and attitude toward the brand. Journal of Electronic Commerce Research, 19(1), 16-35.

Zhang, H., Lu, Y., Gupta, S., \& Zhao, L. (2014). What motivates customers to participate in social commerce? The impact of technological environments and virtual customer experiences. Information \& Management, 51(8), 1017-1030. https://doi.org/10.1016/j.im.2014. 07.005 .

Zhao, K., Stylianou, A. C., \& Zheng, Y. (2018). Sources and impacts of social influence from online anonymous user reviews. Information \& Management, 55(1), 16-30. https://doi.org/10.1016/j.im.2017.03. 006.

Zhou, M. H., Li, P. M., \& Mou, Y. P. (2015). Effects of online reviews on purchase intention of consumers-the mediation of psychological distance. Soft Science, 291, 101-104.

Zhu, D. H., Ye, Z. Q., \& Chang, Y. P. (2017). Understanding the textual content of online customer reviews in B2C websites: a cross-cultural comparison between the US and China. Computers in Human Behavior, 76, 483-493. https://doi.org/10.1016/j.chb.2017.07.045.

Publisher's note Springer Nature remains neutral with regard to jurisdictional claims in published maps and institutional affiliations. 